\title{
Design study of a low-emittance high-repetition rate thermionic rf gun
}

\author{
A. Opanasenko and V. Mytrochenko \\ NSC/KIPT, Academicheskay 1, Kharkiv 61108, Ukraine \\ V. Zhaunerchyk \\ University of Gothenburg, Kemivägen 9, Gothenburg 41296, Sweden \\ V. A. Goryashko* \\ Uppsala University, Lägerhyddsvägen 1, Uppsala 75120, Sweden
}

(Received 13 August 2016; revised manuscript received 19 January 2017; published 31 May 2017)

\begin{abstract}
We propose a novel gridless continuous-wave radiofrequency (rf) thermionic gun capable of generating $\mathrm{nC}$ ns electron bunches with a rms normalized slice emittance close to the thermal level of $0.3 \mathrm{~mm}$ mrad. In order to gate the electron emission, an externally heated thermionic cathode is installed into a stripline-loop conductor. Two high-voltage pulses propagating towards each other in the stripline-loop overlap in the cathode region and create a quasielectrostatic field gating the electron emission. The repetition rate of pulses is variable and can reach up to one $\mathrm{MHz}$ with modern solid-state pulsers. The stripline attached to a rf gun cavity wall has with the wall a common aperture that allows the electrons to be injected into the rf cavity for further acceleration. Thanks to this innovative gridless design, simulations suggest that the bunch emittance is approximately at the thermal level after the bunch injection into the cavity provided that the geometry of the cathode and aperture are properly designed. Specifically, a concave cathode is adopted to imprint an 2-shaped distribution onto the beam transverse phase-space to compensate for an S-shaped beam distribution created by the spherical aberration of the aperture-cavity region. In order to compensate for the energy spread caused by rf fields of the rf gun cavity, a 3rd harmonic cavity is used. A detailed study of the electrodynamics of the stripline and rf gun cavity as well as the beam optics and bunch dynamics are presented.
\end{abstract}

DOI: 10.1103/PhysRevAccelBeams.20.053401

\section{INTRODUCTION}

During the last decade a number of projects and proposals on accelerator-based $\mathrm{x}$-ray Compton sources and other compact sources have been put forward [1]. An essential ingredient of such sources is high-peak-current low-emittance electron bunches with a repetition rate in the range from a $\mathrm{kHz}$ to hundreds of $\mathrm{MHz}$. For pump-probe experiments such as, for instance, $\mathrm{THz}$ pump/x-ray probe of magnetization dynamics [2], the repetition rate must be adjustable depending on the process in question and also match that of the pump and detector. Picosecond (ps) or sub-ps electron bunch duration is another requirement on the electron source crucial for time-resolved studies. Yet one more request from users on the electron beam is the continuous wave $(\mathrm{CW})$ mode of operation with evenly spaced electron bunches [3].

There are technologies for generation of electron beams with the required parameters but these technologies are

\footnotetext{
*vitaliy.goryashko@physics.uu.se; vitgor06@gmail.com

Published by the American Physical Society under the terms of the Creative Commons Attribution 3.0 License. Further distribution of this work must maintain attribution to the author $(s)$ and the published article's title, journal citation, and DOI.
}

rather complicated whereas small laboratories need simple and robust solutions. In this paper, we set forth a novel design of a $\mathrm{CW}$ rf gun with a thermionic cathode that generates a low-emittance beam. In what follows, we give a brief overview of the existing electron gun technologies and point out the place of our design in the field.

High-voltage DC guns with photocathodes [4,5] were the first guns to meet the requirements of extra high bunch quality and high-repetition rate. However, backward ion bombardment leads to photocathode degradation and limits its lifetime [6]. In order to minimize the amount of residual gases and associated ion background, the vacuum in the gun cavity must be extremely high. In addition, the generation of ions by the core and by the halo of electron beams makes problematic to operate an injector at $\mathrm{mACW}$ current levels [7]. One more limitation pertinent to this type of guns is a lower electric breakdown threshold in static fields compared to that in rf fields [8].

At present, superconducting (SC) rf guns with photocathodes [9] generate $\mathrm{CW}$ trains of ultra high-brightness electron bunches. A SC gun is compatible with a high accelerating gradient at moderate requirements for peak $\mathrm{rf}$ power compared to normal conducting counterparts. The bunch charge and energy can be as high as a few $\mathrm{nC}$ and a few $\mathrm{MeV}$, respectively. However, the combination of the 
complexity of the SC technology and bunch formation makes this type of gun very challenging for design and operation. Care must be taken about the cleanness to avoid the cavity contamination. The emittance compensation by a magnetic field penetrating into the cavity is not possible since the field can cause a breakdown of superconductivity.

In view of the challenges with DC and SC photocathode guns, there is a natural interest in extending conventional normal conducting ( $\mathrm{NC}$ ) rf guns to $\mathrm{CW}$ operation. The main obstacle is the heat load so that the power density must be low, which implies a low frequency and field strength of the accelerating mode. Along this line, a $433 \mathrm{MHz}$ Boeing rf gun delivering a few $\mathrm{nC}$, several $\mathrm{mm}$ mrad bunches at $27 \mathrm{MHz}$ repetition rate and $25 \%$ duty factor was demonstrated [10]. Another example of such an approach is the APEX gun $[11,12]$ for modern $\mathrm{x}$-ray free-electron lasers (FELs). However, a short photocathode lifetime [13], for example, only up to 10 hours for the Boeing gun [10], and the need for a high average power drive laser makes this technology also rather complicated for a small facility. Replacement of a photocathode by a thermionic one will further reduce costs and make the gun even easier to operate.

In general, thermionic cathode-based DC and rf guns have successfully been used in IR FELs [14-16] and proved long lifetime at high average currents and modest vacuum. However, because of the grid used to gate the electron emission [17] the mentioned thermionic guns have a large initial transverse emittance of around 10-20 mm mrad. Hence, a gridless thermionic gun generating a $\mu \mathrm{s}$ bunch was proposed for the SACLA FEL [18]. The gating is done by a ns high-voltage (HV) chopper following the gun. Though the bunch quality is sufficient to drive $\mathrm{x}$-ray FELs [19], the repetition rate is limited to $60 \mathrm{~Hz}$.

Recently, in [20,21] a combination of a rf cavity gun with a DC pulsed gate-electrode and a thermionic cathode has been proposed for the XFEL-O injector [22]. However, no details on the cathode assembly were reported. Somewhat earlier, a synthesis of a photoexcited DC $1 \mathrm{GV} / \mathrm{m}$ preaccelerator, directly followed by an S-band rf-booster had been studied [23]. In the spirit of the idea of the combined thermionic DC-rf gun, in this paper we present a detailed design study of a CW normal conducting thermionic rf gun with a dispersion-free configuration of the cathode-gateelectrode region. Given that a two-conductor transmission line allows dispersion-free propagation of transverse electromagnetic (TEM)-modes, our idea is to use a stripline with a thermionic cathode integrated into it so that we can apply high voltage square-box pulses to the cathode-gateelectrode gap with very little distortion. The proposed design occupies a distinguished niche among the thermionic and photo rf guns and has the following features: (i) nanoCoulomb bunch charges; (ii) very low slice emittance thanks to a small-size cathode and careful design of the beam optics; (iii) variable repetition rate of electron bunches up to one $\mathrm{MHz}$ in the present design with the possibility of the repetition rate at $\mathrm{rf}$ frequencies; (iv) long-term stable operation of thermionic cathodes; (v) compatibility with magnetic fields for emittance compensation; (vi) high accelerating gradients of $20 \mathrm{MV} / \mathrm{m}$ in a rf gun cavity.

This paper is organized as follows: in Sec. II we present a layout of the gun and discuss its electrodynamic characteristics; Sec. III contains an analysis of the beam optics in a quasistatic approximation whereas the bunch dynamics in rf fields is studied in Sec. IV; in Sec. V we present the results of fully time-dependent simulations and Sec. V summarizes the findings. Following the terminology of [24], we use the term beam optics for motion of a continuous flow of electrons in static fields and the term bunch dynamics for motion of a group of electrons in time-dependent fields.

\section{LAYOUT AND ELECTRODYNAMICS OF THE GUN}

Figure 1 shows a schematic of the proposed pulse-gated thermionic rf gun. The gun is based on a normal conducting cavity with an operating frequency of $117.4 \mathrm{MHz}$ that corresponds to the third subharmonic of the fundamental accelerating mode of the SC spoke cavity $(352.2 \mathrm{MHz})$ [25]. The choice of the rf gun frequency was motivated by (i) the required electron bunch parameters, (ii) availability of well-developed technology of production of $\mathrm{NC} \mathrm{rf}$ cavities in the very high frequency (VHF) band $[11,26]$,

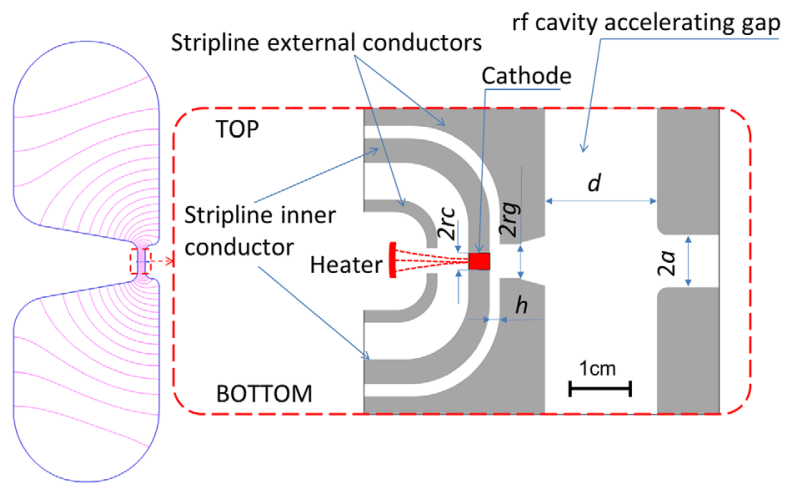

FIG. 1. A schematic of the proposed HV pulse-gated thermionic rf gun. The inset shows the cathode assembly and the on-axis region of an accelerating gun cavity. A cylindrical singlecrystal $\mathrm{CeB}_{6}$ cathode of radius $r_{c}$, schematically shown by the red rectangle, is installed into the central conductor of a striplineloop. The wall of the gun cavity embodies the outer conductor of the stripline-loop so that electron bunches are injected to the gun cavity through an aperture in this part of the outer conductor. The input aperture radius is $r_{g}$ and the radius of the output cavity aperture is $a$. The accelerating gaps of the stripline-loop and cavity are $h$ and $d$, respectively. The cathode can be heated by a DC laser or a diode DC electron gun labeled as the heater in the inset. The dotted red lines represent schematically the rays from the laser heater or the trajectories of electrons emitted by the electron heater. 
and (iii) by availability of efficient $\mathrm{rf}$ sources at this frequency. To power the cathode-gate gap by high voltage rectangular pulses of ns duration without distortion we propose to use a stripline-based thermionic cathode configuration shown in the inset of Fig. 1.

The thermionic cathode is located in the inner conductor of a stripline that is fed by a high voltage fast pulser. The gate-electrode is the external conductor of the stripline and simultaneously part of the rf cavity wall. The gate has a circular aperture-referred to as the input aperture- that allows an electron bunch emitted from the thermionic cathode to flow through the aperture during a $10 \mathrm{MV} / \mathrm{m}$ flattop of a waveform. Then, a $20 \mathrm{MV} / \mathrm{m}$ electric field of the rf gun cavity extracts the electrons and accelerates them up to $450 \mathrm{keV}$. This energy is a trade-off between minimization of the emittance growth due to space-charge effects $[18,27]$ and possibility for further compression of the bunch in a drift space [16].

We chose a single-crystal $\mathrm{CeB}_{6}$ as a material for the thermionic cathode due to its successful operation in the X-ray FEL at SPring-8 [19]. At a temperature of $1773 \mathrm{~K}$ and a field of $10 \mathrm{MV} / \mathrm{m}$ on the cathode, the emission current density is calculated to be $20.5 \mathrm{~A} / \mathrm{cm}^{2}$ that is similar to the results found in the Refs. [20,21]. In this temperaturelimited regime the Richardson constant and effective work function are measured to be $19.1 \mathrm{~A} \mathrm{~cm}^{-2} \mathrm{~K}^{-2}$ and $2.26 \mathrm{~V}$ [18], respectively. The cathode can be heated by a DC lowcurrent electron beam or laser beam illuminating the cathode from the side opposite to the emission surface. To this end, there is an additional aperture in the external conductor of the stripline, see the inset of Fig. 1.

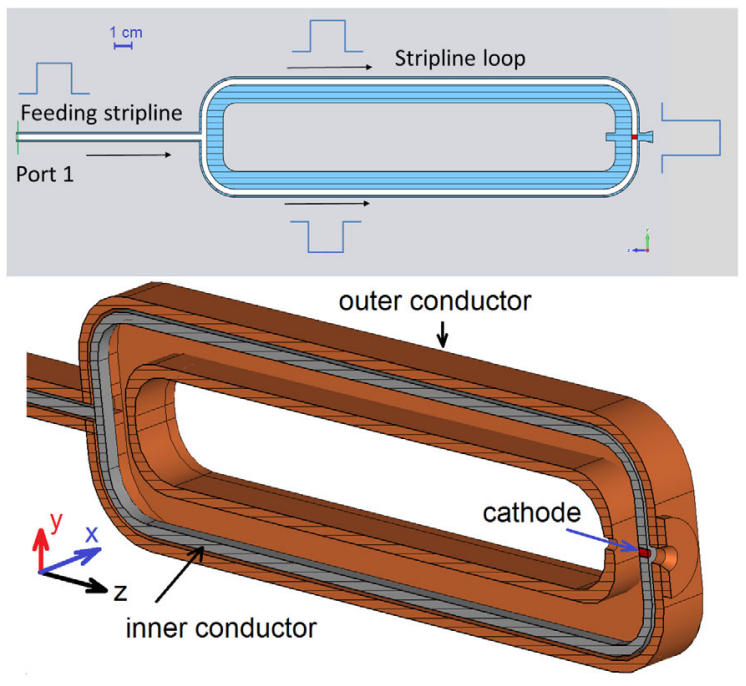

FIG. 2. On top, a 2D cross-sectional-in the electron bunch plane-drawing of the symmetrical stripline-loop based cathode configuration. The inner conductor and vacuum region are depicted in white and blue, respectively. The cathode is schematically shown by the red rectangle located in the inner conductor. On bottom, a cross-sectional drawing of the striplineloop in perspective. (a)

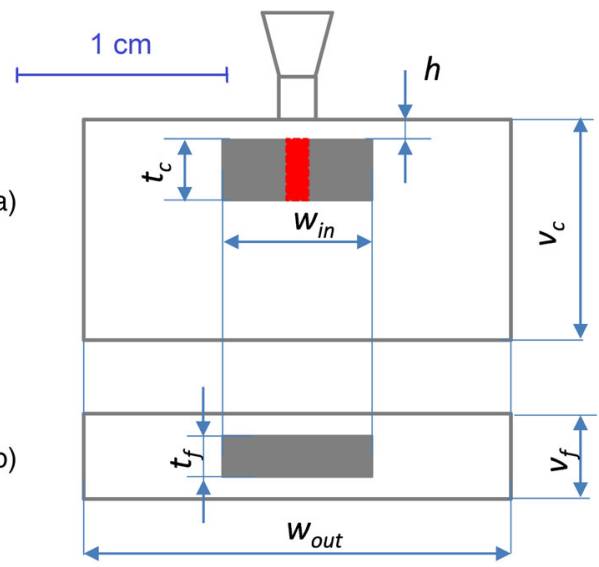

FIG. 3. Two cross-sections of the stripline-loop: (a) plane $x z$ across the cathode, (b) plane $x y$ across the feeding stripline. The gray rectangular areas represent the inner conductor of the stripline-loop (upper plot) and the feeding stripline (bottom plot). The cathode is shown by the red rectangle. In the feeding stripline the position of the inner conductor is symmetrical whereas in the stripline-loop the inner conductor is displaced in order to provide a higher voltage in the cathode-gate-electrode gap.

\section{A. A stripline-based cathode configuration}

In order to avoid the emittance dilution originating from the magnetic field on the cathode, we propose to gate the electron emission by two TEM electromagnetic pulses propagating towards each other so that on the cathode the electric fields sum up in phase whereas the magnetic fields of pulses cancel each other. Basically, one obtains a quasistatic electric field in the region of the cathode-gate-electrode during the pulse. The cathode is installed in a striplinemade of an inner and outer metallic conductor with vacuum in between-forming a closed loop as it is depicted in Fig. 2. The stripline-loop can be thought as a T-junction power divider with the arms connected to each other. It is a parallel connection so that the voltage in each arm is the same as in the feeding line whereas the current is half of the value before splitting. To reach a high electric field in the region of the cathode-gate-electrode, the inner conductor is displaced towards the part of the outer conductor surface attached to the gun cavity wall, see Fig. 3(a). Matching of the feeding stripline to the stripline-loop is achieved by means of adjustment of the thickness of the inner conductor and

TABLE I. Parameters of the stripline-loop in Fig. 3(a).

\begin{tabular}{lcc}
\hline \hline Parameter & Symbol & Value \\
\hline Cathode-gate-electrode gap & $h$ & $1 \mathrm{~mm}$ \\
Inner conductor thickness & $t_{c}$ & $3 \mathrm{~mm}$ \\
Inner conductor width & $w_{\text {in }}$ & $7 \mathrm{~mm}$ \\
Outer conductor height & $v_{c}$ & $13 \mathrm{~mm}$ \\
Outer conductor width & $w_{\text {out }}$ & $20 \mathrm{~mm}$ \\
TEM mode impedance & $Z_{\text {loop }}$ & $31.72 \Omega$ \\
Peak voltage at the cathode & $U_{\mathrm{c}}$ & $17.86 \mathrm{kV}$ \\
\hline \hline
\end{tabular}


TABLE II. Parameters of the feeding stripline in Fig. 3(b).

\begin{tabular}{lcc}
\hline \hline Parameter & Symbol & Value \\
\hline Inner conductor thickness & $t_{f}$ & $3 \mathrm{~mm}$ \\
Inner conductor width & $w_{\text {in }}$ & $7 \mathrm{~mm}$ \\
Outer conductor height & $v_{f}$ & $4.52 \mathrm{~mm}$ \\
Outer conductor width & $w_{\text {out }}$ & $20 \mathrm{~mm}$ \\
TEM mode impedance & $Z_{\text {feed }}$ & $15.86 \Omega$ \\
Peak voltage in the line & $U_{1}$ & $9 \mathrm{kV}$ \\
\hline \hline
\end{tabular}

the height of the external conductor. The parameters of the striplines are summarized in Tables I and II.

Performance of the stripline loop in the time domain is illustrated in Fig. 4 using the simulations done with the CST Microwave Studio [28]. A trapezoidal high-voltage (HV) pulse in the form of the TEM mode is supplied to the feeding stripline, then equally split between the arms and combined within the cathode region. A transmitted pulse comes out of the stripline-loop with little distortion as it is seen from the blue curve in Fig. 4. Even without careful optimization of the T-junction a relative peak-to-peak disturbance of the flattop of the transmitted pulse is around $2 \%$. The considered pulse can be generated by a fast solid-state HV pulser, see, for example, [29]. Due to the transverse nature of the TEM stripline mode and symmetry of the stripline-loop, the magnetic fields on the cathode cancel while the electric ones add up in phase as illustrated in Fig. 5. Doubling of the voltage in the cathode-gate-electrode gap allows one to halve the feed power from a fast pulser compared to the case of feeding by a single pulse. For our geometry a $10 \mathrm{MV} / \mathrm{m}$ electric field on the cathode corresponds to a voltage of $17.86 \mathrm{kV}$ in the cathode-electrode gap. This requires a $9 \mathrm{kV}$ pulser with an average power of $5.11 \mathrm{~kW}$ for a pulse length of $1 \mathrm{~ns}$ at one $\mathrm{MHz}$ repetition rate.

\section{B. Fields in the cathode region}

Figure 6 shows the electric field profiles on the cathode surface (see the inset in Fig. 1) along two mutually perpendicular axes. The origin of the bunch axis corresponds to $x=y=0$. In the cathode region for radii up to $2.2 \mathrm{~mm}$ the field has azimuthal symmetry to a large extent. A field drop across the $1 \mathrm{~mm}$ cathode radius is due to a relatively large gate aperture of $r_{g}=2 \mathrm{~mm}$ compared with

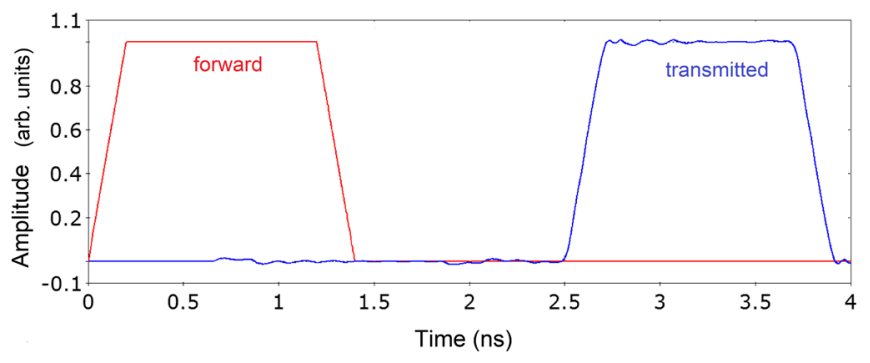

FIG. 4. The forward and transmitted pulses at the feeding port. the $1 \mathrm{~mm}$ cathode-gate-electrode gap. Because of the observed symmetry of the electromagnetic (EM) field in the cathode region and the azimuthal symmetry of the rf gun cavity, in what follows we use a $2 D$ approach for studying the beam optics and dynamics.

Figure 7 shows a set of distributions of the longitudinal electric field $E_{z}(x, z)$ for different $z$ as function of $x$. Recall

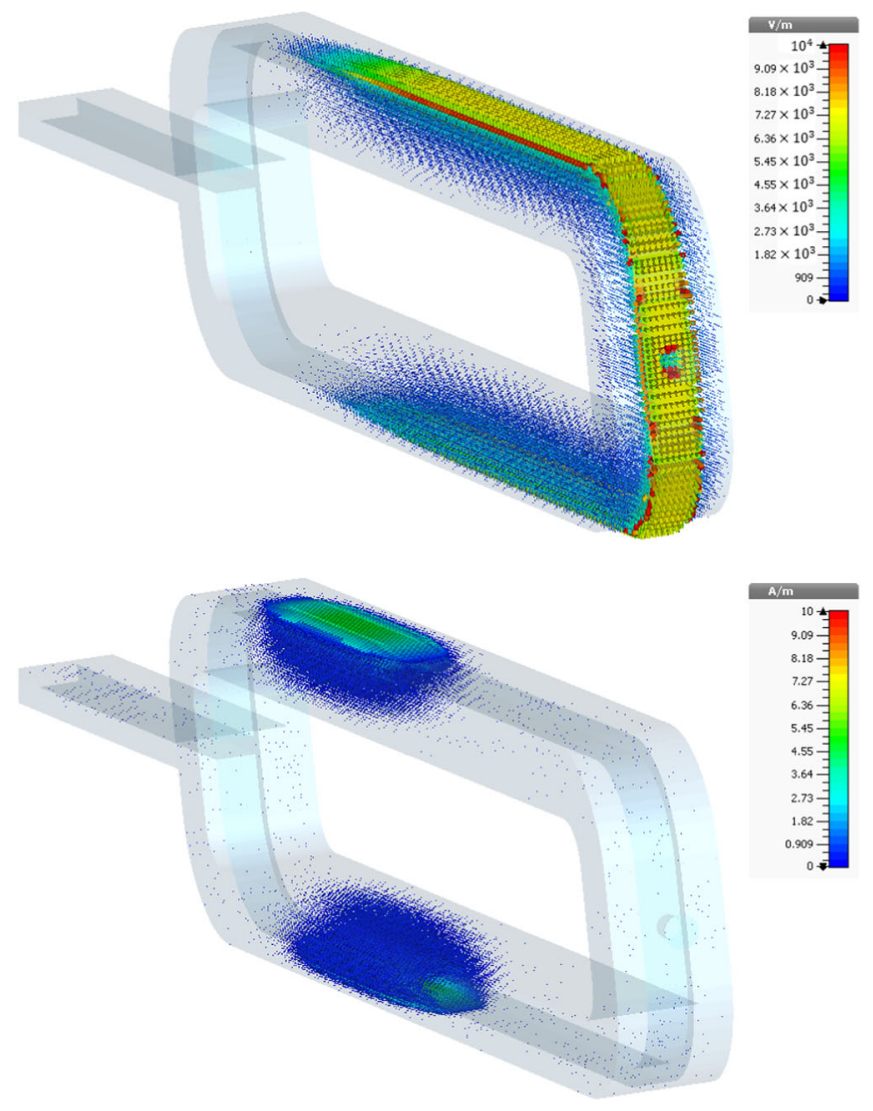

FIG. 5. The electric (top) and magnetic (bottom) field distributions in the stripline-loop at the moment when the middle of the pulse passes the cathode. The magnetic fields cancel each other. The simulation is performed with the CST microwave studio.

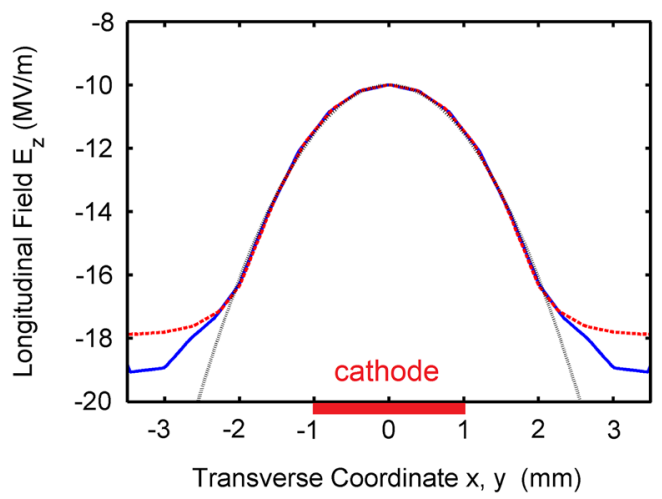

FIG. 6. The electric field profiles on the cathode surface along (red) and across (blue) the inner conductor. The dotted black curve represents the analytical approximation (1). 


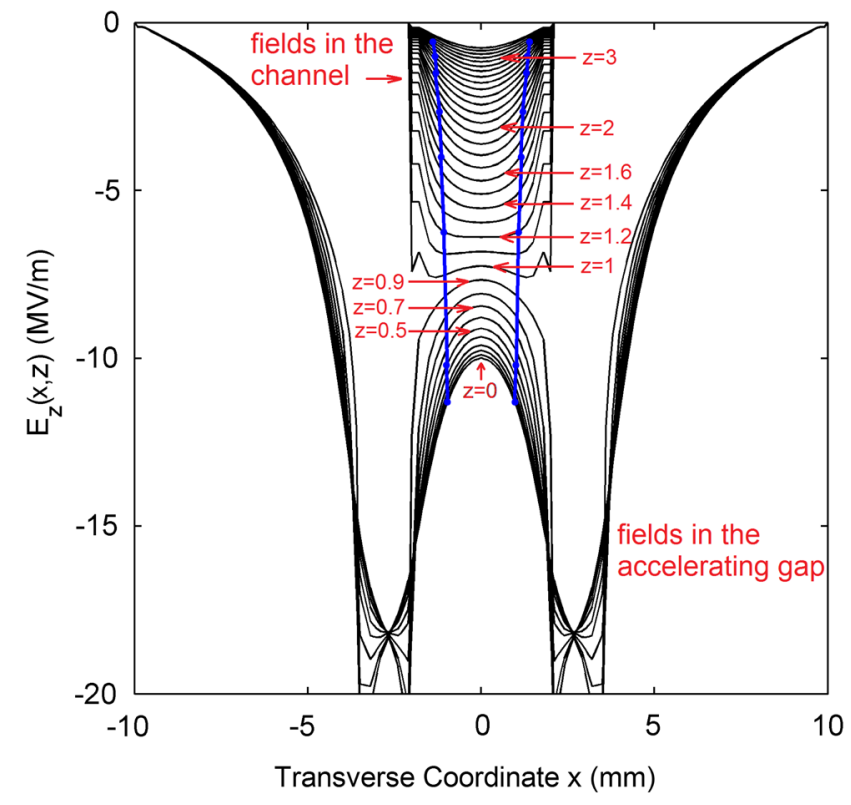

FIG. 7. A family of curves (black) of the longitudinal electric field $E_{z}(x, z)$ for different $z$ as a function of $x$. The field is shown in the cross section orthogonal to the conductors of the striplineloop and drawn through the cathode, see the upper drawing in Fig. 3. For $z<1$ the field extends over the region from $-10 \mathrm{~mm}$ to $10 \mathrm{~mm}$, which corresponds to the interior space of the striplineloop, whereas for $z \geq 1$ the field is limited to the space inside the aperture channel. The blue curves depict the envelope of the electron beam.

that $z=1 \mathrm{~mm}$ corresponds to the edge of the aperture channel. In the vicinity of the beam axis $x=0$, the magnitude of $E_{z}(x, z)$ in the accelerating gap $(z<1)$ changes with $x$ as the modified Bessel function of the first kind, which is a natural representation for an unbounded space electrostatic problem, whereas in the channel of the aperture $E_{z}(x, z)$ behaves as the standard Bessel function of the first kind of a bounded-space electrodynamic problem. It is interesting to see how the sign of the curvature of $E_{z}(x, z)$ changes as one moves into the channel. A virtual interface between different behaviors of $E_{z}(x, z)$ with $x$ is approximately located at $z_{0}=1.2 \mathrm{~mm}$. In Fig. 7 we have also depicted (blue curves) the envelope of the electron beam discussed in detail later.

The distribution depicted in Fig. 7 suggest the following phenomenological expression for the longitudinal field in the region $r<r_{g}$ :

$$
E_{z}(r, z)=E_{0} J_{0}\left[\mu_{01} \frac{r}{r_{g}} \sqrt{\frac{z-z_{0}}{r_{g}}}\right] \exp \left[-\left(\frac{z}{2 h}\right)^{2}\right] .
$$

Because of the symmetry pointed out earlier, we use the cylindrical coordinates $r$ and $z$. Equation (1) can be understood as follows. The last multiplier takes into account the longitudinal localization of the field on the scale of $2 h$ [30]. For $z \gg h$, i.e., deeply inside the aperture channel, the radial distribution of the field must mimic that of a waveguide mode, so the second multiplier $J_{0}[\ldots]$ is the Bessel function of the first kind with $\mu_{01} / r_{g}$ being an eigenvalue of the Laplace equation [31]. But for $z<h$, the radial distribution must manifest the character of an electrostatic solution, so the argument of $J_{0}[\ldots]$ incorporates the square root of $(z-h)$ that yields the transformation to the modified Bessel function upon transition through $z=z_{0}$. The difference $(z-h)$ is naturally normalized to the scale of the transverse inhomogeneity $r_{g}$. The field amplitude $E_{0}$ and transition position $z_{0}$ must be determined from numerical simulations. A detailed comparison of the simple representation (1) with the numerical solution depicted in Fig. 7 shows as low peak-to-peak discrepancy as $10 \%$, which allows using Eq. (1) for an analytical study of beam optics. The analytical fit (1) can be made nearly perfect on the cathode surface if $r_{g}$ is adjusted to $2.4 \mathrm{~mm}$.

\section{Rf gun cavity}

The considered rf gun cavity is similar to re-entrant cavities used in klystrons. A design of a rf cavity and an analysis of the bunch dynamics are mutually coupled: the targeted bunch parameters impose requirements on the cavity geometry (and accelerating fields) as well as the geometry of the input and output apertures for bunch passage. At the same time, the geometry affects the bunch dynamics. We use successive iterations to address this complicated coupled dynamic system. First, we performed a tentative study of the rf gun cavity, then designed the input and output apertures taking into account the beam optics and lastly studied the bunch dynamics in rf fields of the cavity. Finally, we refined

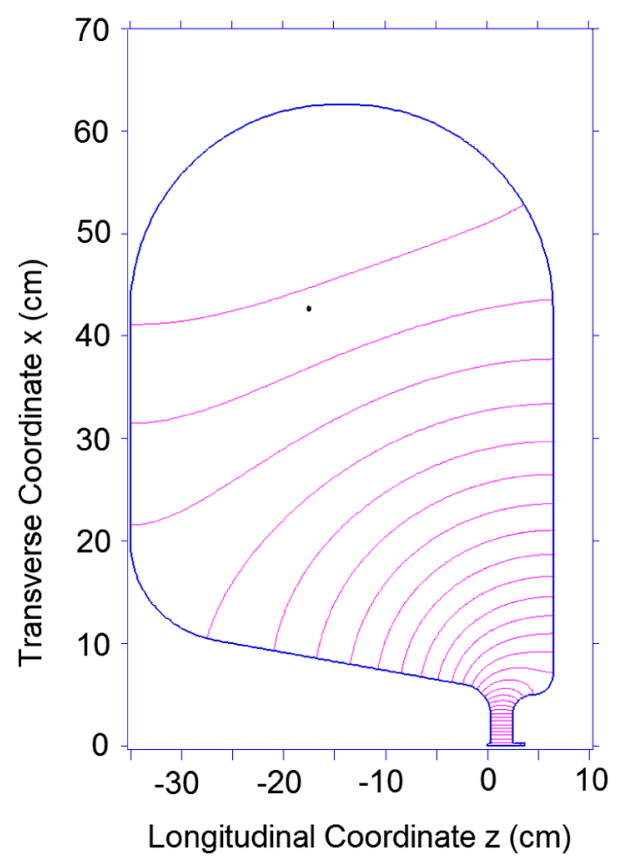

FIG. 8. The geometry and electric field distribution in the rf gun cavity designed with SUPERFISH. 
TABLE III. The main electrodynamic parameters of the designed rf gun cavity.

\begin{tabular}{ll}
\hline \hline Parameter & \multicolumn{1}{c}{ Value } \\
\hline Frequency & $117.4 \mathrm{MHz}$ \\
Nominal gradient & $20 \mathrm{MV} / \mathrm{m}$ \\
Accelerating potential & $430 \mathrm{kV}$ \\
Accelerating gap & $21 \mathrm{~mm}$ \\
Power dissipation & $16.9 \mathrm{~kW}$ \\
Stored energy & $1.13 \mathrm{~J}$ \\
Shunt impedance & $299.6 \mathrm{M} \Omega / \mathrm{m}$ \\
Quality factor & 49284 \\
$E_{\max } / E_{0}$ & 2.0 \\
$B_{\max } / E_{\max }$ & $0.326 \mathrm{mT} /(\mathrm{MV} / \mathrm{m})$ \\
\hline \hline
\end{tabular}

the geometry of the rf gun cavity and repeated the bunch dynamics simulations. Here, we present the final results. Rf couplers are not included into the design.

Our design goals of the cavity are a high shunt impedance along with small ratios $B_{\max } / E_{\max }$ and $E_{\max } / E_{0}$ in order increase the rf breakdown threshold of the cavity. Here, $B_{\max }$ and $E_{\max }$ are the maximum surface magnetic and electric fields, respectively; $E_{0}$ is the accelerating gradient defined as the average on-axis electric field. As a starting point for our design we chose the geometry of the CW NC VHF gun cavity proposed at Argonne [11,32]. The resulting cavity shape obtained with SUPERFISH [33] is similar to that of [11] and depicted in Fig. 8. Since the cavity is azimuthally symmetric with respect to the bunch axis, only a half of the cross section is shown. The main parameters of the cavity are summarized in Table III and the on-axis profile of the longitudinal electric field is shown in Fig. 9. In order to be able to study the rf gun cavity and cathode region independently, in the simulations we enforced an electric wall boundary condition-i.e., the tangential electric field vanishes-located at $z=3.5 \mathrm{~mm}$ from the cathode, which is approximately in the middle of the input aperture. The position of the electric wall is determined by a superposition of rf fields and fields of HV pulses in the cathode-gate-electron gap. This is discussed in detail in the

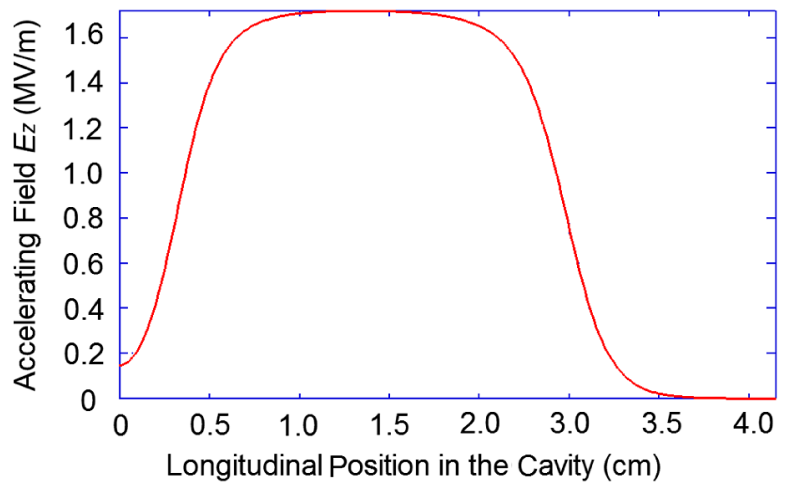

FIG. 9. The longitudinal electric field profile along the accelerating gap of the gun cavity. The accelerating potential is $36.533 \mathrm{kV}$. next section. As a result of this additional boundary condition, the longitudinal electric field starts from a nonzero value in Fig. 9. The field to the left from the zero position is determined by the HV pulser as well as the geometry of cathode region and aperture.

\section{BEAM OPTICS}

\section{A. Electrostatic approach}

Quasi-square-shaped HV pulses produced by modern solid-state pulsers have a stable flattop region, see Ref. [29] and references therein. Hence, electrons emitted from the cathode experience a quasistatic electric field in the cathode-gate-electrode gap and their trajectories may be examined in an electrostatic approximation using the simulation code EGUN [34,35]. In addition to that, the electrostatic approximation may also be extended to the $r f$ gun cavity to perform a tentative study of the beam optics in the whole gun. Thanks to the low frequency of the rf gun cavity, the $140 \mathrm{ps}$ transit time of electrons through the cavity is much smaller than the $8.5 \mathrm{~ns}$ rf period and a slice of electrons experiences the same rf field. Hence, the rf field that can be replaced by a corresponding accelerating DC voltage. Moreover, the electron bunch is much longer than the interaction region so that it fills the whole gun and may be thought as a continuous beam (edge effects are addressed in Sec. V). An advantage of the electrostatic approach is the possibility to perform efficient optimization of the shape of both the cathode and input as well as output apertures in the cavity wall.

The radius of the gate-electrode aperture, its profile and thickness were adjusted to avoid beam losses and maximally suppress the penetration of the rf cavity field through the aperture into the cathode-gate-electrode space where the field from the HV pulser should be dominant. This optimization resulted in the shape of the input aperture depicted in Fig. 10. The trajectories of rays of electrons and equipotential lines of the electric field are also shown. The

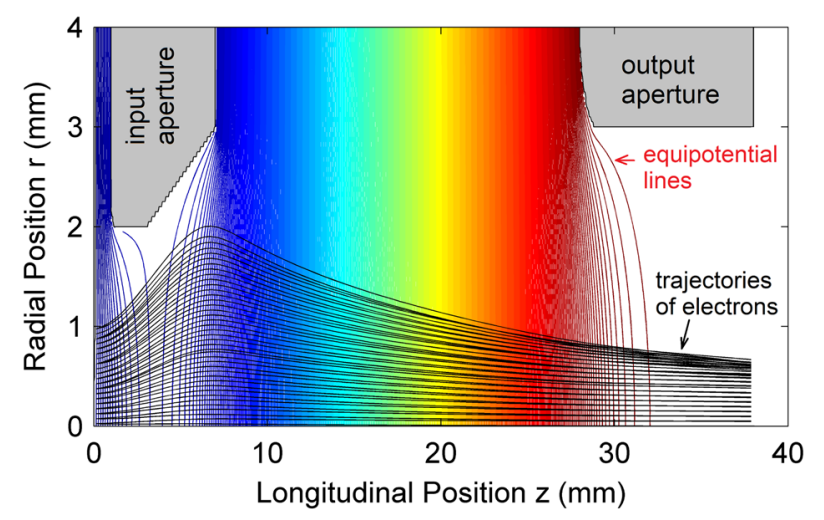

FIG. 10. The gun geometry used in EGUNsimulations. The electrostatic equipotential lines are coded by color such that dark blue and dark red correspond to the maximum negative and zero potential, respectively. 
TABLE IV. The parameters of the paraxial region of the gun.

\begin{tabular}{lcc}
\hline \hline Parameter & Symbol & Value \\
\hline Cathode-gate-electrode gap & $h$ & $1 \mathrm{~mm}$ \\
Radius of gate-electrode aperture & $r_{g}$ & $2 \mathrm{~mm}$ \\
Cathode radius & $r_{c}$ & $1 \mathrm{~mm}$ \\
rf gun cavity accelerating gap & $d$ & $21 \mathrm{~mm}$ \\
Radius of output cavity aperture & $a$ & $3 \mathrm{~mm}$ \\
\hline \hline
\end{tabular}

main parameters are given in Table IV. In the simulations we considered the gate and anode potentials to be 17.86 and $473 \mathrm{kV}$, respectively. In this model, the far wall of the cavity plays a role of the anode. These potentials correspond to electric field strengths of $10 \mathrm{MV} / \mathrm{m}$ on the cathode and $20 \mathrm{MV} / \mathrm{m}$ in the cavity, respectively. Given the cathode radius of $1 \mathrm{~mm}$ and emission current of $21.2 \mathrm{~A} / \mathrm{cm}^{2}$, the beam current is $0.665 \mathrm{~A}$ that results in a total charge of around $0.7 \mathrm{nC}$ for an $\mathrm{HV}$ pulse with $1 \mathrm{~ns}$ flattop. At the cathode the simulated rms normalized transverse beam emittance is $0.28 \mathrm{~mm}$ mrad that is just slightly above the theoretical value of the thermal emittance of $0.5 r_{c} \sqrt{k_{b} T_{c} / m}=0.27 \mathrm{~mm}$ mrad at $1773 \mathrm{~K}$. Here, $k_{b}$ is the Boltzmann constant and $T_{c}$ is the cathode temperature.

Figure 11 depicts the longitudinal electric field profile along the gun axis at a gate potential of $17.86 \mathrm{kV}$ and two anode potentials of $473 \mathrm{kV}$ and $410 \mathrm{kV}$. These field profiles in the gun cavity represent the instantaneous distributions of the rf fields seen by a test electron accelerated on-crest and $21.13^{\circ}$ off-crest. In the cross section $z=3.5 \mathrm{~mm}$ of the gate aperture, the amplitude of the electric field reaches a minimum. To the left from this cross section, the electric field of the HV pulser is dominant and constant during the pulse whereas to the right from the cross section, the field is solely determined by the rf cavity field. In other words, the

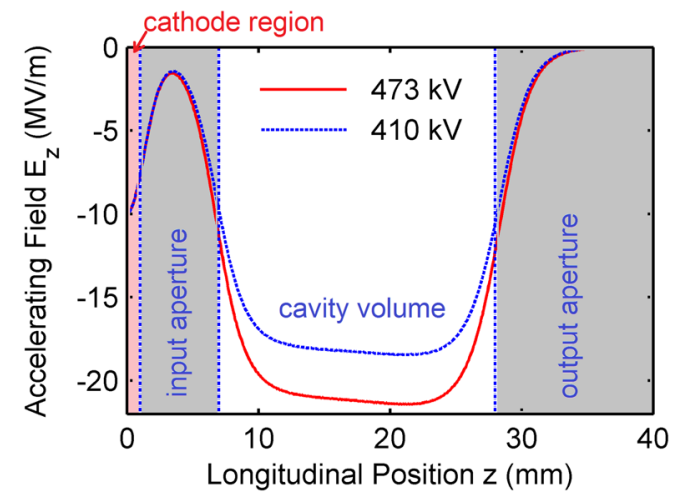

FIG. 11. The profile of the longitudinal electric field in the gun vs. distance. The simulation is perform with EGUNfor the whole interaction region from the cathode, $z=0 \mathrm{~mm}$, to the end of output aperture of the cavity, $z=38 \mathrm{~mm}$. The electric field on the cathode produced by the $\mathrm{HV}$ pulser is $10 \mathrm{MV} / \mathrm{m}$. The aperture region extends from $z=1 \mathrm{~mm}$ to $z=7 \mathrm{~mm}$, where the absolute value of the field has an extremum. region around the cathode is well shielded from cavity rf fields and beam acceleration is completely determined by the electrostatic field of the HV pulser. Furthermore, the radial component of the external electric field vanishes in this cross section since it is proportional to the derivative of the longitudinal component that has an extremum at that point. Hence, the cross section can be thought of as an electric wall for external fields in electromagnetic simulations. We use this fact to decouple the study of beam optics from bunch dynamics. First, we apply the EGUN code to the whole gun and optimize the beam optics, then in the position of the electric wall we transfer the obtained beam data to the PARMELA code and continue phase-dependent beam dynamics simulations in the rf gun cavity with PARMELA. The space-charge effect is included in both simulations. The purpose of using EGUN is that it takes accurately into account image charges in the stripline-loop. The rf magnetic field is not included into the EGUN simulation because the magnetic force is negligibly small compared to the electric one. Specifically, the cavity magnetic field is related to the electric one as $B^{\mathrm{rf}} \sim$ $(r / \lambda) E^{\mathrm{rf}}$ with $\lambda$ being the $\mathrm{rf}$ wavelength. The $\mathrm{rf}$ field decays exponentially into the aperture channel as $\mathrm{e}^{-\mu_{01} z / r_{g}}$ and, in addition, the beam is weakly-relativistic. Hence, the ratio of forces $F_{B}^{\mathrm{rf}} / F_{E}^{\text {static }} \sim 10^{-6}$ in the stripline loop and $F_{B}^{\mathrm{rf}} / F_{E}^{\text {static }} \sim 10^{-3}$ in the position of the introduced electric wall. In PARMELA simulations, the magnetic field is naturally included.

In Fig. 12 we demonstrate the change of the beam emittance and envelope along the gun from the cathode to the output aperture of the rf cavity. The off-axis profile of the radial electric field causing an emittance growth is shown as well. This profile suggests that the cathodeaperture region and aperture-cavity region can respectively be thought of as a defocusing and focusing lens so that the

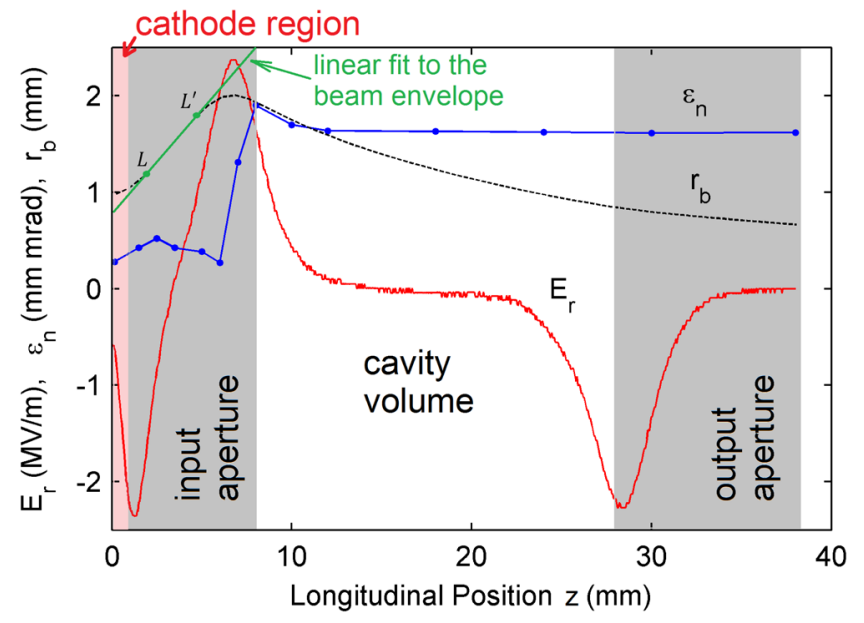

FIG. 12. The normalized rms emittance in mm mmrad (blue curve with dots), the beam envelop in $\mathrm{mm}$ (dotted black) and radial electric field distribution at $r=1 \mathrm{~mm}$ in $\mathrm{MV} / \mathrm{m}$ (solid red) vs. the longitudinal position. The results are obtained with EGUN. 
beam first expands reaching up to $2 \mathrm{~mm}$ at the end of the $6 \mathrm{~mm}$ thick input aperture. Then, the focusing effect takes over and the beam radius becomes less than $1 \mathrm{~mm}$ in the output cavity aperture. One can also see a 6-fold increase in emittance at the exit of the input aperture caused by the spherical aberration [24]. Note that there is a little increase in emittance in the regions of the rf cavity and output aperture because in the cavity the transverse electric field is almost zero and when the beam traverses the output aperture it has a much higher energy and a smaller radius. From the analytical estimate presented below, it follows that the nonlinear emittance growth is proportional to the fourth power of the beam radius so that the reduction of it by a factor of two in the output aperture-compared to that in the input one-implies more than an order of magnitude decrease in emittance growth.

\section{B. Spherical aberration of the cathode-aperture region}

Examine in detail the beam characteristics in the region from $z=0$ to $z=3.5 \mathrm{~mm}$, where the accelerating field of the stripline-loop is dominant, Fig. 10. By scrutinizing Fig. 12, one finds that over the initial distance of $2.5 \mathrm{~mm}$ the rms radius increases by $30 \%$ and the emittance almost doubles reaching $0.51 \mathrm{~mm}$ mrad. Then, the emittance declines to $0.42 \mathrm{~mm}$ mrad as the beam undergoes further action mainly from the stripline-loop field. As we will see this behavior of the emittance is a manifestation of the nonlinear electrostatic emittance compensation demonstrated in [36].

In general, the origin of emittance growth is the nonlinearity of the accelerating field of the stripline-loop as well as the space-charge field. To understand the impact of the former consider a laminar nonrelativistic beam of rays of electrons in the cathode-aperture region. The beam moves in a potential field of the stripline-loop so that the total and longitudinal velocities read

$$
v(r, z)=\sqrt{\frac{2 e}{m} V(r, z)}, \quad v_{z}(r, z)=\sqrt{\frac{2 e}{m} V_{b}(r, z)}
$$

with $V=\int_{C} \vec{E}\left(\vec{r}^{\prime}\right) \cdot d \vec{r}^{\prime}$ and $V_{b}=\int_{0}^{z} E_{z}\left(r, z^{\prime}\right) d z^{\prime}$ being the accelerating voltages along some path $C$ and the $z$-axis, respectively. Here, $\vec{r}^{\prime}=(r, z)$ is the radius-vector in the cylindrical coordinates, $e$ and $m$ are the charge and mass of the electron, respectively. The maximum value of $V(r, z)$ is the difference of potentials between the cathode and gate electrode (cavity wall) $V_{0}$. The conservation of energy yields the radial velocity in the form

$$
v_{r}=\sqrt{\frac{2 e}{m}\left[V(r, z)-V_{b}(r, z)\right]} .
$$

To account for the beam expansion, the analysis must be complemented with an equation for radial positions of the rays of electrons $d r_{e} / d z=v_{r} / v_{z}$. In general, $r_{e}$ is a nonlinear function of $z$ and an initial position of the ray $r_{0}$. To get a glimpse of the formation of the beam phase-space, let us examine the case of straight rays of electrons [37]. At the end of the accelerating region, where the field vanishes, the beam velocity is simply $v=\sqrt{(2|e| / m) V_{0}}$ and in the paraxial approximation $v_{r}$ can be simplified to

$$
v_{r}=r \sqrt{\frac{e V_{b}^{\prime \prime}}{m}}\left(1+\frac{r^{2}}{24} \frac{V_{b}^{\prime \prime \prime \prime}}{V_{b}^{\prime \prime}}\right) .
$$

Here, $V_{b}^{\prime \prime}=\partial^{2} V_{b} / \partial r^{2}$ and $V_{b}^{\prime \prime \prime \prime}=\partial^{4} V_{b} / \partial r^{4}$. Using Eq. (1), we obtain the radial beam divergence as

$$
\begin{aligned}
\frac{v_{r}}{v_{z}} & =\frac{\mu_{01} r_{0}}{2 r_{g}} \sqrt{g}\left[1+\frac{g}{2}\left(\frac{\mu_{01} r_{0}}{4 r_{g}}\right)^{2}\left(1+\frac{2 h^{2}(\pi-2)}{g^{2} \pi r_{g}^{2}}\right)\right], \\
g & =\frac{z_{0}}{h}-\frac{2 h}{r_{g} \sqrt{\pi}} .
\end{aligned}
$$

Equation (5) gives the beam divergence at the end of the defocusing lens but it is also instructive to look into individual contributions to the divergence from the fields in the accelerating gap and aperture channel. In Fig. 13 we depicted effective deflecting voltages in the accelerating stripline gap (dotted blue curve) defined as $\left[r^{2} \int_{0}^{z_{0}} E_{z}^{\prime \prime}\left(0, z^{\prime}\right) d z^{\prime}+r^{4} \int_{0}^{z_{0}} E_{z}^{\prime \prime \prime \prime}\left(0, z^{\prime}\right) d z^{\prime}\right]$, in the aperture channel (dashed black curve) defined as $\left[r^{2} \int_{z_{0}}^{\infty} E_{z}^{\prime \prime}\left(0, z^{\prime}\right) d z^{\prime}+r^{4} \int_{z_{0}}^{\infty} E_{z}^{\prime \prime \prime \prime}\left(0, z^{\prime}\right) d z^{\prime}\right]$, and the sum of the two (solid red curve). Because of the different signs of the curvature of $E_{z}(r, z)$, see Fig. 7, the deflecting voltage in the aperture channel partly compensates that one of the stripline gap. For straight rays of electrons this compensation works only for the quadratic components of the voltages whereas the quartic components add up. This implies compensation of the linear in $r$ component of $v_{r}$ since $v_{r} \propto \sqrt{V_{b}}$. Hence, Eq. (5) can be thought of as the worst-case estimate for emittance growth. Specifically, a calculation based on Eq. (5) gives an emittance growth of $0.3 \mathrm{~mm} \mathrm{mrad}$.

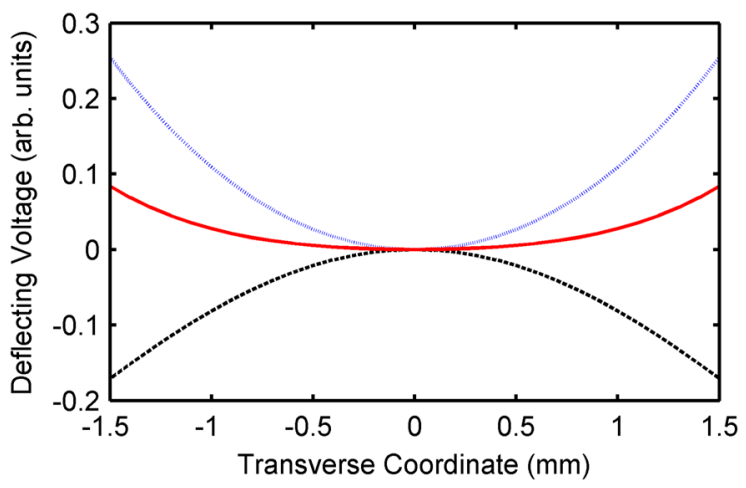

FIG. 13. Gain in the deflecting voltage in the accelerating stripline gap (dotted blue curve), the aperture channel (dashed black curve) and the sum of the two (solid red curve) vs transverse coordinate. 


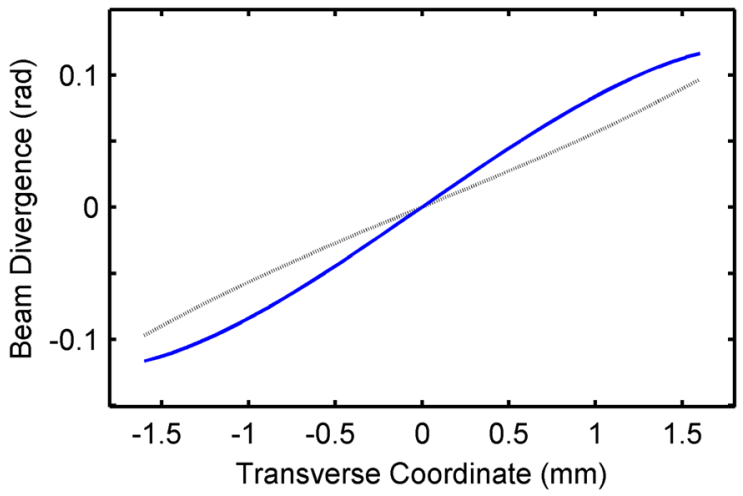

FIG. 14. Phase-space of a beam of straight rays (dotted black) and a realistic beam (solid blue) at $z=3.5 \mathrm{~mm}$.

In a realistic case, however, the rays of electrons are curved as depicted in Fig. 10 and our analytical calculations for the curved rays revealed also the possibility of selfcompensation of the quartic component of the deflecting voltage. Namely, in the region from $z=0$ to $z=1.2 \mathrm{~mm}$ the stripline-loop field imprints an Z-shape distribution onto the transverse beam phase-space whereas in the region from $z=1.2$ to $z=3.5 \mathrm{~mm}$ it imprints an S-shape distribution. The physical origin of this difference is connected to a change of the sign of the field distribution curvature, see Fig. 7. Depending on the rate of the beam expansion, the beam phase-space distributions acquired in different regions balance each other to certain extent. Moreover, S.B. van der Geer and others demonstrated that a nonlinear electrostatic accelerating field can even compensate for the space-charge effect [36], which imprints an 2-shape distribution. In Fig. 14 we depicted the results of analytical calculations of the phase-space of a beam of straight rays and a realistic beam at $z=3.5 \mathrm{~mm}$.

Performing simulations with and without taking into account the space-charge field, we found that its inclusion leads to a non-negligible emittance dilution. However, the space-charge field affects the beam mainly in a close vicinity to the cathode as one can see from Fig. 15. Beyond that region the space-charge field becomes small. For example, in the region of the virtual electric wall, where the external field vanishes, the rays of electrons are almost perfectly straight as seen from Fig. 10. This indicates a negligibly small transverse acceleration. An impact of the space-charge field on the overall gun performance is addressed at the end of this section.

\section{Spherical aberration of the aperture-cavity region}

As we have already pointed out, there is a huge emittance growth at the end of the aperture channel. To address this growth within an analytical model, we need an approach different to the one we used above since the beam becomes relativistic. A radial momentum kick experienced by a test electron traversing the aperture reads
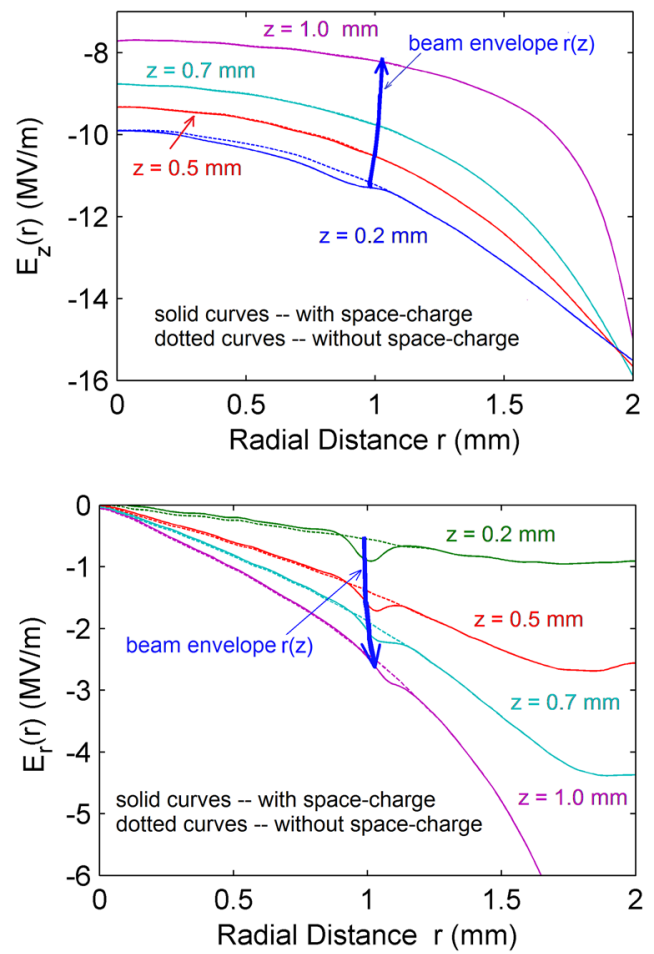

FIG. 15. A set of distributions of the longitudinal (upper plot) and transverse (bottom plot) electric field as function of the radial distance for different longitudinal positions. The solid curves depict solutions to the Poisson equation whereas the dotted curves are solutions to the Laplace equation. The beam envelope with the arrow pointing out the direction of increasing $z$ is also depicted.

$$
\Delta p_{r}=e \int_{z_{\text {in }}}^{z_{\text {out }}} \frac{e \tilde{E}_{r}\left(r, z^{\prime}\right)}{v_{z}\left(z^{\prime}\right)} d z^{\prime}
$$

where $\tilde{E}_{r}$ is the radial electric field in the aperture-cavity region (we use the $\tilde{E}$ to distinguish this field from the stripline-loop field $E$ ); $z_{\text {in }}$ and $z_{\text {out }}$ are the coordinates of the start and end of the electric field "jump," which roughly corresponds to the middle and exit planes of the aperture. In the paraxial approximation using Maxwell's equations the radial component of the electric field can be expressed through the longitudinal one as

$$
\begin{aligned}
& \tilde{E}_{r}(r, z) \approx-\frac{r}{2} \frac{\partial}{\partial z}\left[\tilde{E}_{z}(0, z)+\frac{r^{2}}{4} \tilde{E}_{z}^{\prime \prime}(0, z)\right], \\
& \left.\tilde{E}_{z}^{\prime \prime}(0, z) \equiv \frac{\partial^{2} \tilde{E}_{z}(r, z)}{\partial r^{2}}\right|_{r=0} .
\end{aligned}
$$

Substituting $\tilde{E}_{r}$ and integrating Eq. (6) once by parts, we derive

$$
\Delta p_{r}=f\left[\tilde{E}_{z}\right] r+f\left[\tilde{E}_{z}^{\prime \prime}\right] r^{3} / 4
$$

where 


$$
f[Y]=-\frac{e}{2}\left[\frac{Y\left(z_{\text {out }}\right)}{v_{z}\left(z_{\text {out }}\right)}-\frac{Y\left(z_{\text {in }}\right)}{v_{z}\left(z_{\text {in }}\right)}+\int_{v_{z}\left(z_{\text {in }}\right)}^{v_{z}\left(z_{\text {out }}\right)} Y \frac{d v_{z}}{v_{z}^{2}}\right] .
$$

The cubic term in $\Delta p_{r}$, which is referred to as $\left[\Delta p_{r}\right]_{3}$ in what follows, gives rise to an emittance growth.

We cannot treat the aperture as a thin electrostatic lens because the beam velocity changes significantly across it. This makes an analysis of the integral in Eq. (9) rather complicated and we limit ourselves to an order-of-magnitude estimate of the nonlinear kick. Our numerical simulations suggest the following approximation for $\tilde{E}_{z}^{\prime \prime}$ :

$$
\tilde{E}_{z}^{\prime \prime} \approx \frac{1}{2}\left(\frac{\mu_{01}}{R}\right)^{2}\left[\tilde{E}_{z}\left(0, z_{\text {out }}\right) U\left(z-z_{m}\right)-\tilde{E}_{z}(0, z)\right],
$$

where $U(z)$ is the unit step function; $z_{m}$ is the position of the maximum of $\tilde{E}_{r}$, see Fig. 12; $R$ is the mean radius of the aperture, which is different from $r_{g}$ because the output side of the aperture is tapered. Substituting Eq. (10) into the integral in Eq. (9), we obtain

$$
\begin{aligned}
\int_{v_{z}\left(z_{\text {in }}\right)}^{v_{z}\left(z_{\text {out }}\right)} \tilde{E}_{z}^{\prime \prime} \frac{d v_{z}}{v_{z}^{2}}= & \frac{1}{2}\left(\frac{\mu_{01}}{R}\right)^{2}\left[-\int_{v_{z}\left(z_{\text {in }}\right)}^{v_{z}\left(z_{\text {out }}\right)} \tilde{E}_{z} \frac{d v_{z}}{v_{z}^{2}}\right. \\
& \left.+\tilde{E}_{z}\left(0, z_{\text {out }}\right)\left(\frac{1}{v_{z}\left(z_{0}\right)}-\frac{1}{v_{z}\left(z_{\text {out }}\right)}\right)\right] .
\end{aligned}
$$

To estimate the remaining integral, we replace the integrand by the mean values. The resulting emittance growth defined as

$$
\Delta \epsilon_{n} \approx \frac{r_{b}\left[\Delta p_{r}\right]_{3}}{2 m c}
$$

takes the form

$$
\begin{aligned}
\Delta \epsilon_{n} \approx & \frac{e r_{b}^{4}}{32 m e}\left(\frac{\mu_{01}}{R}\right)^{2}\left[\frac{\tilde{E}_{z}\left(z_{\text {out }}\right)}{v_{z}\left(z_{\text {out }}\right)}\left(\frac{1}{v_{z}\left(z_{0}\right)}-\frac{1}{v_{z}\left(z_{\text {out }}\right)}\right)\right. \\
& \left.+\frac{\tilde{E}_{z}\left(z_{\text {in }}\right)}{v_{z}\left(z_{\text {in }}\right)}-\frac{\tilde{E}_{z}\left(z_{\text {in }}\right)+\tilde{E}_{z}\left(z_{\text {out }}\right)}{2} \frac{\delta v_{z}}{\bar{v}_{z}^{2}}\right],
\end{aligned}
$$

where $\delta v_{z}$ is the change in velocity. Consider a numerical example: (i) the field jump is from $\tilde{E}_{z}\left(z_{\text {in }}\right)=1.2 \mathrm{MV} / \mathrm{m}$ to $\tilde{E}_{z}\left(z_{\text {out }}\right)=20.5 \mathrm{MV} / \mathrm{m}$; (ii) the beam velocity varies from $v_{z}\left(z_{\text {in }}\right)=0.258 c$ to $v_{z}\left(z_{\text {out }}\right)=0.534 c$ with the mean of $\bar{v}_{z}=0.337 c, v_{z}\left(z_{0}\right) \approx \bar{v}_{z}$; (iii) the geometrical parameters are $R=2.55 \mathrm{~mm}$ and $r_{b}=1.95 \mathrm{~mm}$. Equation (13) gives an emittance growth of $\Delta \epsilon_{n}=$ $1.55 \mathrm{~mm} \mathrm{mrad}$ that is together with the emittance before the aperture $\epsilon_{n}\left(z_{\text {in }}\right)=0.428 \mathrm{~mm}$ mrad yields the total emittance of $\epsilon_{n}\left(z_{\text {out }}\right)=\sqrt{\epsilon_{n}\left(z_{\text {in }}\right)^{2}+\Delta \epsilon_{n}^{2}} \approx 1.61 \mathrm{mmmrad}$. This semianalytical estimate predicts well the $1.7 \mathrm{~mm}$ mrad emittance obtained with EGUN. From results (8)-(11) it follows that this spherical aberration gives rise to a classical

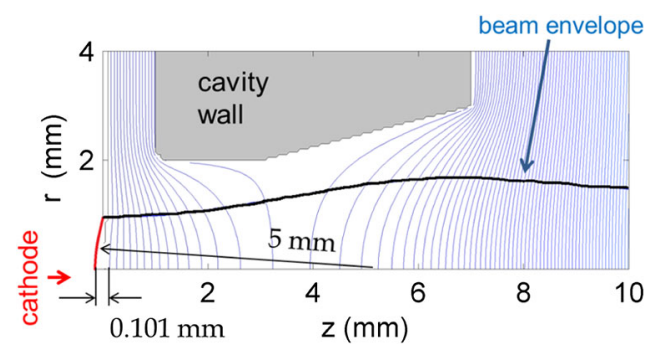

FIG. 16. Gun geometry with the optimized cathode profile.

S-shape distribution of the transverse phase-space of the beam [24].

\section{Elimination of emittance growth}

To reduce the emittance growth caused by the spherical aberration in the aperture-cavity region, we made the cathode concave. The introduced sphericity of the cathode creates transverse momenta of electrons that depend on the electron's radial position and cancel quite well the transverse nonlinear kicks caused by the aperture. The maximum rms beam radius also gets smaller by $15 \%$. The geometry of the gun with a profiled cathode is shown in Fig. 16. A comparison of the emittance variation along the gun with a flat and concave cathode is presented in Fig. 17. When the cathode shape is properly chosen, the beam emittance-after a large initial growth-approaches the thermal emittance level at the end of the input aperture and increases only slightly in the rf cavity. The simulation predicts it to be even slightly below the thermal level but we attribute this to numerical errors.

To understand the physics of the emittance compensation, consider the beam phase-space beside the concave cathode. The geometry of the problem is illustrated in Fig. 18. We will follow the lines of Shintake's analysis of emittance dilution due to surface roughness of a cathode

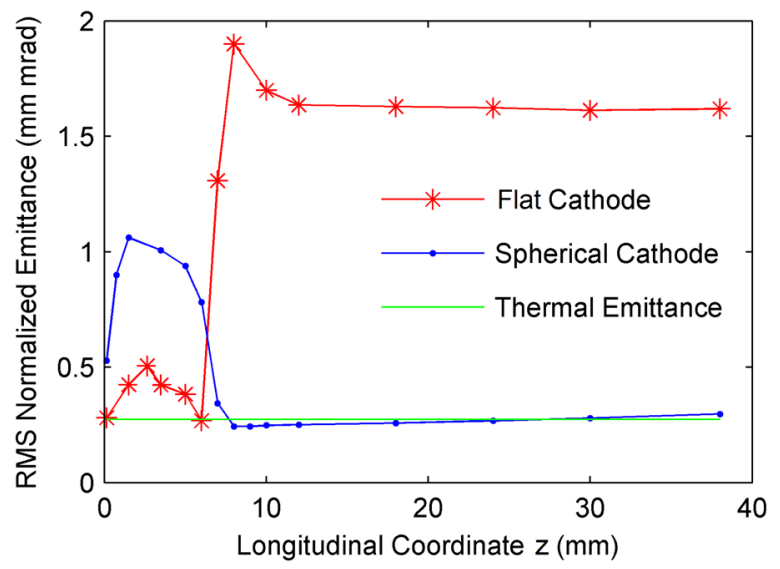

FIG. 17. The normalized rms emittance in mm mmrad for a flat and concave cathode vs. the longitudinal distance. The thermal emittance is shown as a reference. 


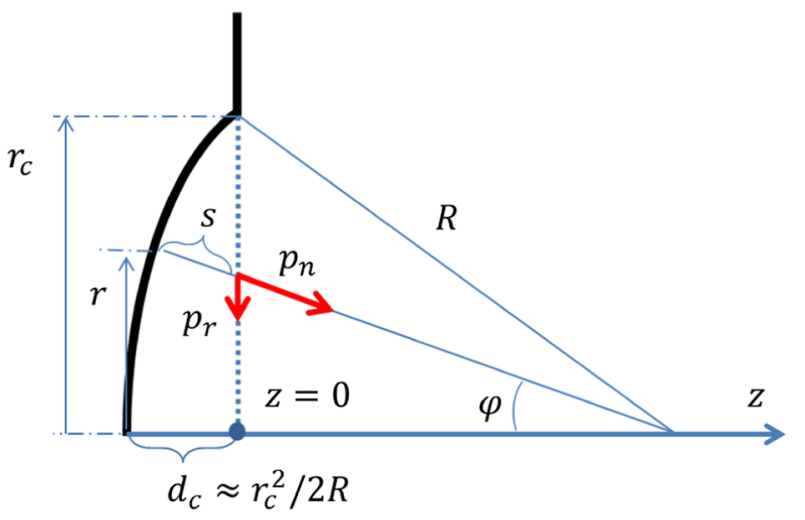

FIG. 18. Beam optics in the vicinity of a concave cathode.

[38]. In the close vicinity of the cathode, the radial and normal components of the electric field are related as $E_{r}=E_{n} \sin \varphi$. Hence, the radial momentum can be estimated as $p_{r}=-p_{n} \sin \varphi \approx-(r / R) p_{n}$. The energy conservation yields $p_{n}=\sqrt{2 m e E_{c} s}$, where $E_{c}$ is the field on the cathode and $s=R-\sqrt{r^{2}+R^{2}-r_{c}^{2}} \approx d_{c}\left(1-r^{2} / r_{c}^{2}\right)$ is the travel distance of the electron emitted at position $r$. Here, $d_{c}=r_{c}^{2} / 2 R$ is the distance between the planes drawn through the apex and edge of the cathode. For estimates, $E_{c}$ can be taken equal to Eq. (1). Moreover, we assumed $E_{c}$ to be constant over $d_{c}$. Then, the radial momentum reads

$$
p_{r}=-\sqrt{2 m e E_{z} d_{c}} \frac{r}{R} \sqrt{1-\frac{r^{2}}{r_{c}^{2}}} .
$$

A comparison of the distributions of the radial momenta calculated analytically and simulated with EGUN is shown in Fig. 19 and demonstrates a good agreement. One can see that the sphericity of the cathode creates a highly nonlinear distribution in the transverse phase-space of the beam. We want to emphasize that the distribution has an Z-shape.

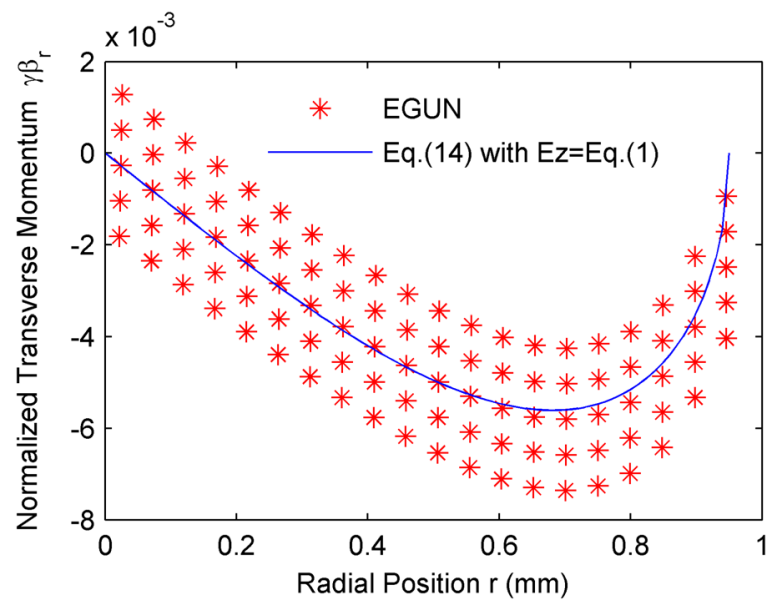

FIG. 19. Transverse phase-space of a beam next to the concave cathode. The curve and dots stand for the analytical and numerical results, respectively.
An emittance growth originating from the sphericity of the cathode reads

$$
\Delta \epsilon_{c}=6.1 \sqrt{\frac{e E_{z} d_{c}}{m c^{2}}} \frac{r_{c}^{2}}{R}
$$

The numerical factor is the result of averaging over the beam phase space. Equation (15) gives the emittance directly in $\mathrm{mm}$ mrad units. With a radius of curvature $R$ of $5 \mathrm{~mm}$, Eq. (15) gives $0.38 \mathrm{~mm} \mathrm{mrad}$, which is together with the initial thermal emittance of $0.28 \mathrm{~mm} \mathrm{mrad}$ results in the total emittance $0.47 \mathrm{~mm}$ mrad. The corresponding result of numerical simulations is $0.51 \mathrm{~mm}$ mrad.

Let us examine the beam phase-space for the flat and concave cathode at $z=3.5 \mathrm{~mm}$ and $z=8 \mathrm{~mm}$. The results of numerical simulations are depicted in Figs. 20, 21, 22, and 23. Recall that the first position $z=3.5 \mathrm{~mm}$ corresponds to the plane, where the radial electric field vanishes, so we refer to this plane as the electric wall. The second position $z=8 \mathrm{~mm}$ is slightly outside of the output plane of the aperture channel connecting the stripline-loop with the gun cavity. In all figures $x^{\prime}=d x / d z$ and $y^{\prime}=d y / d z$ is the angular divergence in the $x$ and $y$ direction, respectively. The bottom left and right plots depict a beam cross section and a relative energy-phase distribution (longitudinal phase-space).

As we found in Sec. III B, the spherical aberration in the region of the cathode-aperture is weak and the spacecharge field is also not strong. Hence, at the electric wall interface the $x x^{\prime}$ and $y y^{\prime}$ phase-space exhibit a nearly linear correlation demonstrated on the top plots in Fig. 20. A careful examination shows that, in fact, the $x x^{\prime}$ distribution has an Z-shape, which is hampered from seeing by energy spread of electrons. We attribute this shape to the spacecharge effect.

In the region of the aperture-cavity electrons acquire the transverse momentum kick (6) that depends nonlinearly on the electron's position in the beam. This results in a deformation of the transverse phase-space manifested as an S-shaped correlation in the $x x^{\prime}$ and $y y^{\prime}$ space, Fig. 21. The correlated energy spread also appears because off-axis electrons experience a different accelerating voltage in the gun cavity.

In the case of the concave cathode, the phase-space dynamics is modified by the transverse momenta (14) so that the transverse phase-space $x x^{\prime}$ and $y y^{\prime}$ at the electric wall interface has an Z-shape distribution shown in Fig. 22. The sphericity of the cathode introduces a correlation into the beam phase-space that is opposite to the correlation imprinted onto the beam by the spherical aberration of the electric focusing lens of the aperture-cavity region. Due to the cancellation of the correlations, after the aperture the transverse phase-space is nearly linear as it is depicted in Fig. 23. Thus, the emittance is greatly reduced. It should be 

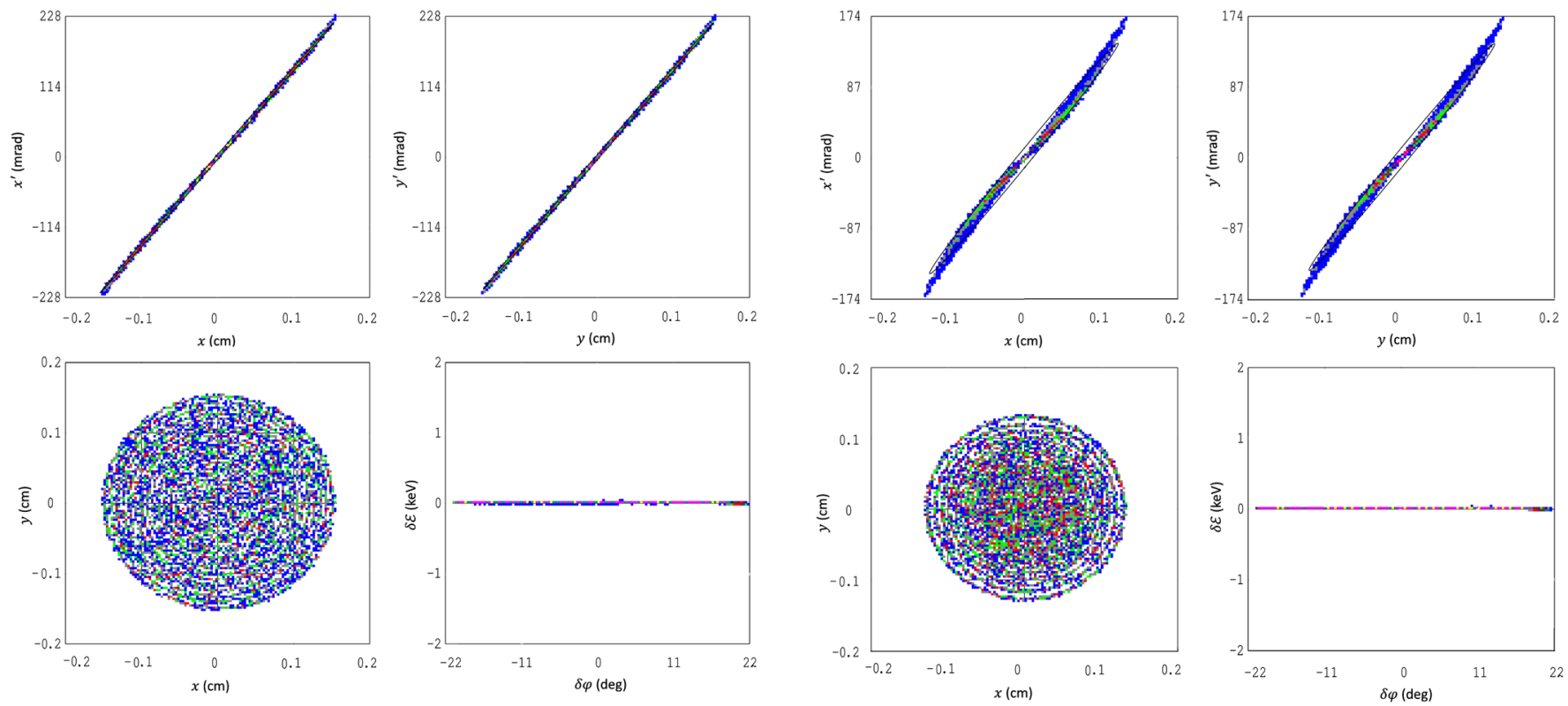

FIG. 20. Beam phase-space in the plane of the electric wall of the input aperture. The cathode is flat; the emittance is $0.423 \mathrm{~mm}$ mrad.

mentioned that at the aperture exit the phase correlation for the flat cathode, Fig. 21, is clearly much stronger than the opposite phase correlation for the concave cathode, Fig. 22. One might wonder why the compensation works. An important additional factor is that the beam size is also different, so electrons emitted by the concave cathode acquire a smaller nonlinear kick in aperture-cavity region compared to the one shown in Fig. 21.
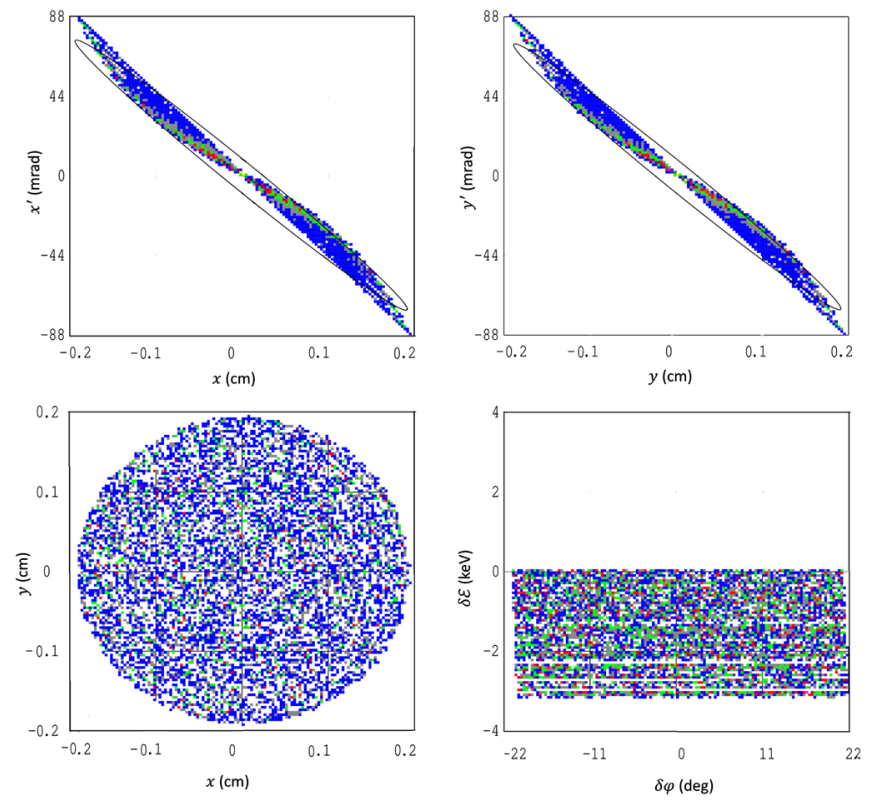

FIG. 21. Beam phase-space in the exit plane of the input aperture. The cathode is flat; the emittance is $1.9 \mathrm{~mm} \mathrm{mrad}$.

FIG. 22. Beam phase-space in the plane of the electric wall of the input aperture. The cathode is concave; the emittance is $1 \mathrm{~mm}$ mrad.

\section{E. Space-charge effects}

Figure 24 shows the dependence of the emittance on the beam current for a flat and a concave cathode. The results are depicted for the positions just after the cathode and at the gun exit. Since time-dependent effects are not included, this should be treated as the best possible result. For the flat cathode, an increase in beam current results in emittance
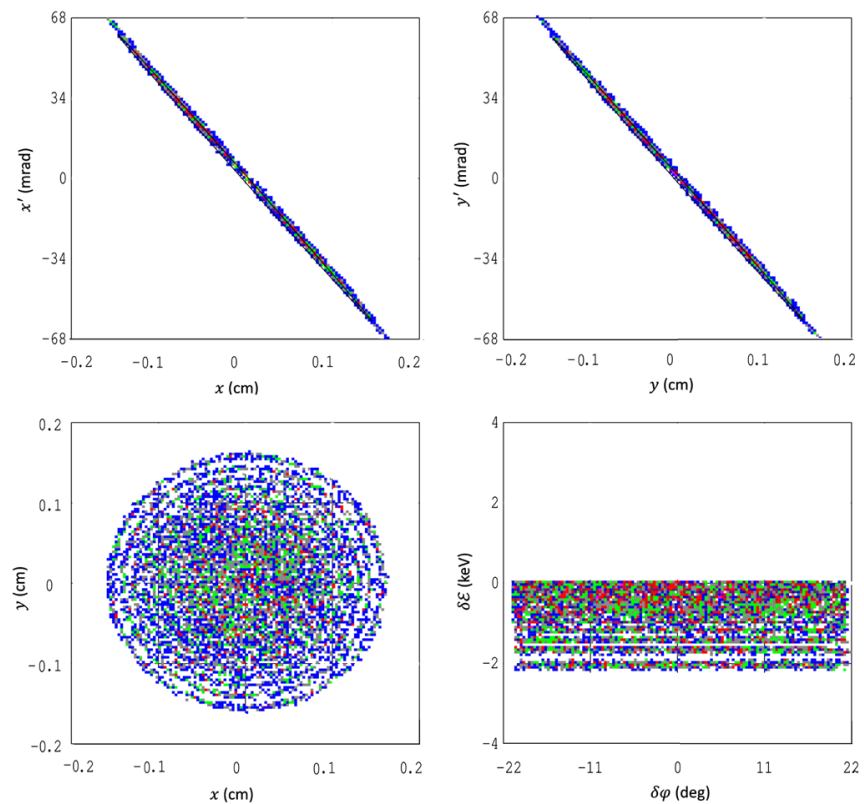

FIG. 23. Beam phase-space in the exit plane of the input aperture. The cathode is concave; the emittance is $0.243 \mathrm{~mm}$ mrad. 


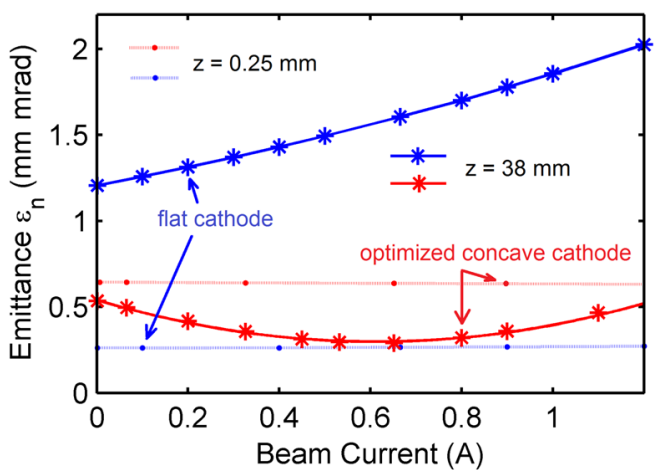

FIG. 24. Emittance vs. current for a flat and concave cathode.

degradation. The situation is completely different for the concave cathode. Thanks to the cathode shaping, the emittance attains its minimum for $0.65 \mathrm{~A}$ beam current and the dependence on the beam current is rather weak so that some tunability of the gun is possible. For instance, a variation of the beam current by $50 \%$ around its optimal value yields only $6 \%$ increase in the beam emittance. Note that the mechanism of emittance compensation in our case is different from the one proposed in Ref. [23]. Specifically, De Loos et al. used the effect of the spherical aberration of the aperture of a DC diode gun to compensate for the deformation of the beam transverse phase-space caused by the space-charge effect. In our case, a concave cathode is used to imprint an Z-shaped distribution onto the beam transverse phase-space to compensate for an S-shaped beam distribution created by the spherical aberration of the aperture-cavity region. With no space-charge field, our compensation would work for any beam current but the space-charge field creates an additional Z-shaped distribution of transverse momenta and also changes the beam radius. Therefore, the emittance is current-dependent.

\section{BUNCH DYNAMICS IN RF FIELDS}

To study the time-dependent effects on the finiteduration electron bunch arising from the rf field of the cavity, we use the code PARMELA. In the longitudinal position $z=3.5 \mathrm{~mm}$, where the radial component of the total electric field vanishes, the bunch parameters are transferred from EGUN to PARMELA and the simulation is continued with the latter. Specifically, in the defined above injection plane the electron bunch has a charge of $650 \mathrm{pC}$ and duration of $1 \mathrm{~ns}$ that corresponds to the initial phase length of $42.26 \mathrm{deg}$ at the cavity frequency of $117.4 \mathrm{MHz}$. The bunch energy and rms normalized emittance are $17.85 \mathrm{keV}$ and $1 \mathrm{~mm}$ mrad, respectively. The bunch is accelerated on-crest in the $21 \mathrm{~mm}$ accelerating gap at a gradient of $20.20 \mathrm{MV} / \mathrm{m}$. This brings the maximum bunch energy to $0.448 \mathrm{MeV}$. During the acceleration the rf curvature is imprinted onto the longitudinal phase-space of the bunch as one can see from the bottom right plot in Fig. 25. The introduced phasedependent energy spread is $2 \%$ but this spread is a correlated
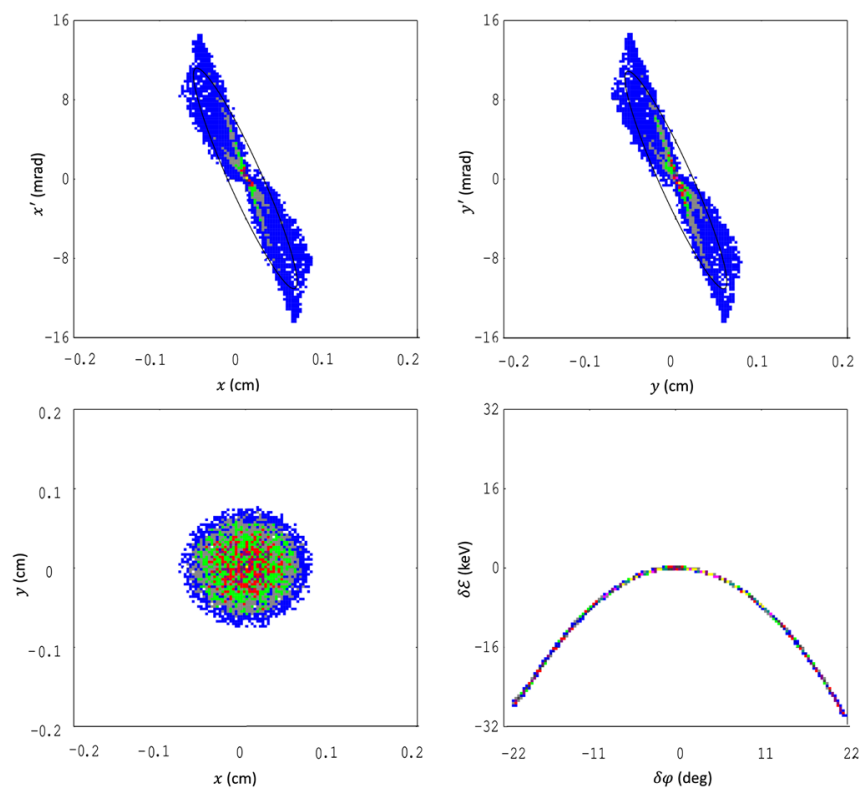

FIG. 25. The bunch phase-space at the output of the rf gun cavity. The cathode is concave.

one so that it is reversible. The bunch emittance also increases up to $0.9 \mathrm{~mm}$ mrad compared to the steady-state result of $0.3 \mathrm{~mm}$ mrad obtained with EGUN. At the same time, the slice emittance remains almost unchanged.

In order to illustrate an advantage of the cathode shaping and also an impact of the time-varying rf field on the beam emittance, in Fig. 26 we present a comparison of the
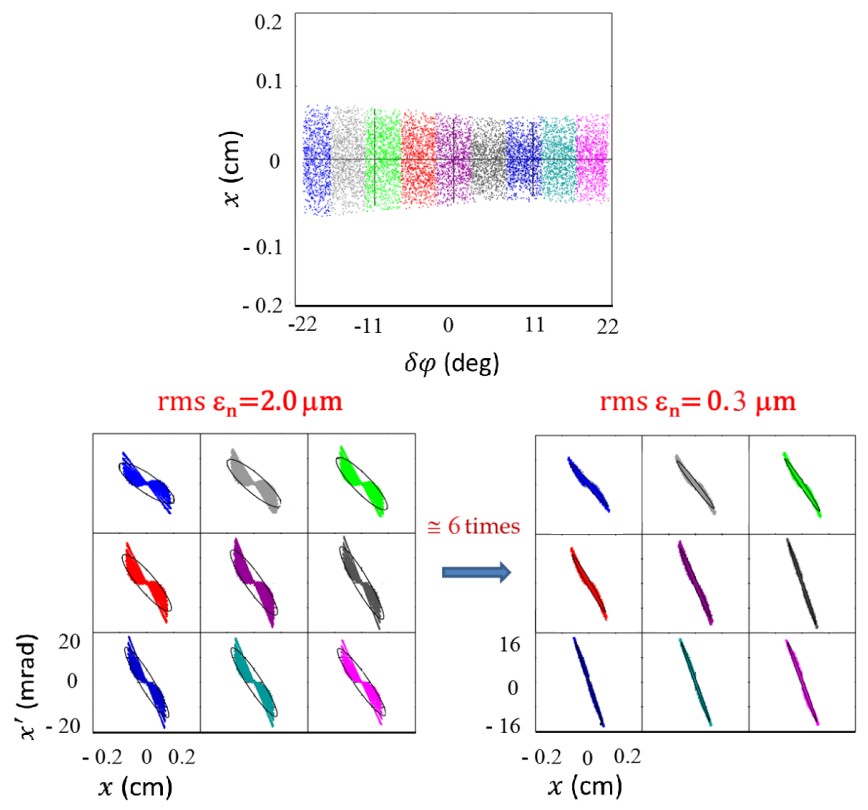

FIG. 26. A comparison of the transverse phase-spaces of the bunch for the flat (bottom left) and concave (bottom right) cathodes at the end of the rf gun cavity. The bunch distribution (top) is represented by 9 consecutive slices along the bunch marked by different colors. 

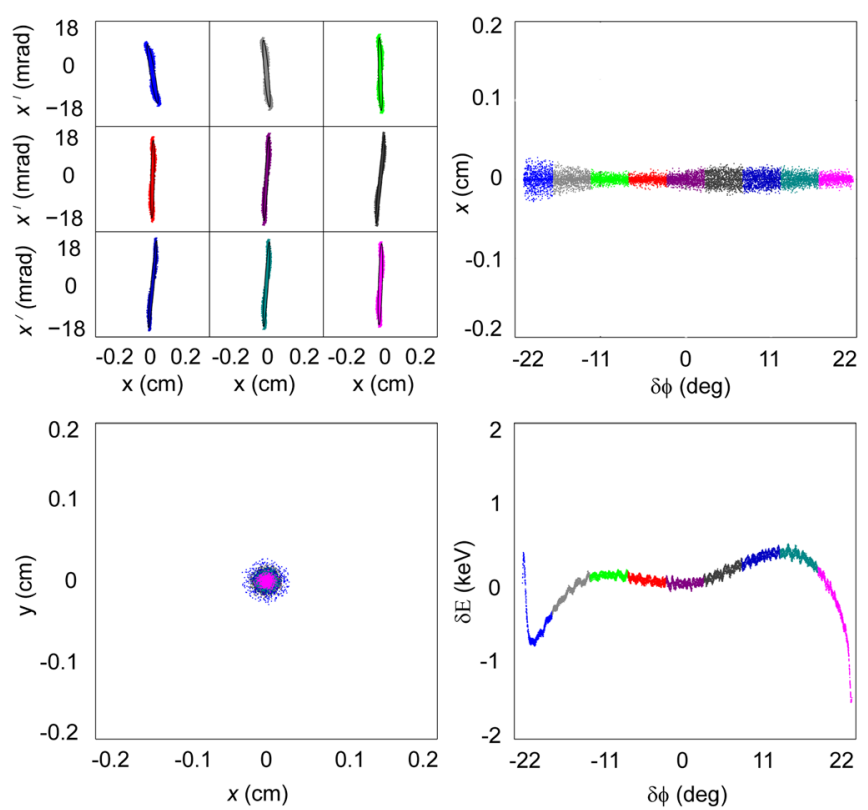

FIG. 27. The bunch phase-space at the output of the 3rd harmonic cavity. Top left: transverse phase-space of slices, top-right: transverse position of electrons vs rf phase, bottom left: transverse distribution of particles; bottom right: longitudinal phase-space.

phase-spaces of the longitudinal slices of the beam. Each slice is just 4.7 deg long so that the electrons in the slice experience approximately the same rf field and the transverse phase-space is represented by a thin ellipse. But because the slices "see" different mean phases of the rf field, they are slightly rotated with respect to each other in the phase-space [39].

In order to counteract the energy spread introduced by the phase-dependent rf field of the gun cavity, we use a third harmonic cavity that acts as a phase-space linearizer [40]. The results are depicted in Fig. 27. The rms energy spread is reduced from 8.8 to $0.48 \mathrm{keV}$ and the energy distribution is substantially flattened for almost the whole bunch. The transverse phase-space distributions $x x^{\prime}$ and $y y^{\prime}$ (not shown) are aligned along the $x^{\prime}$ and $y^{\prime}$ axes, respectively, which correspond to a bunch crossover with a radius of $0.3 \mathrm{~mm}$. The transverse density distribution recovers

TABLE V. The bunch parameters at the output of the 3rd harmonic cavity.

\begin{tabular}{ll}
\hline \hline Parameter & \multicolumn{1}{c}{ Value } \\
\hline Charge & $650 \mathrm{pC}$ \\
Duration & $1 \mathrm{~ns}$ \\
Mean energy & $398 \mathrm{keV}$ \\
Relative rms energy spread & $0.1 \%$ \\
rms normalized emittance & $0.6 \mathrm{~mm} \mathrm{mrad}$ \\
rms normalized slice emittance & $0.3 \mathrm{~mm} \mathrm{mrad}$ \\
Maximum radius & $0.3 \mathrm{~mm}$ \\
\hline \hline
\end{tabular}

very well its initial homogeneous top-hat shape. Note that not only the longitudinal phase-space is linearized but also the emittance growth acquired in the rf gun cavity is partly compensated by the rf field of the 3rd harmonic cavity. The final bunch parameters are summarized in Table V.

\section{TEMPORAL EVOLUTION OF THE ELECTRON BUNCH DISTRIBUTION}

To analyze the beam behavior in the cathode-aperture region of the stripline-loop configuration, we used the static approximation, which allowed us to greatly reduce the complexity of the problem and to address the design of beam optics separately from bunch dynamics. However, now we apply a fully time-dependent approach to study the buildup and decay of space-charge fields as well as the start-to-end evolution of the electron bunch distribution. Specifically, we use the code PARMELA with a 3D particlein-cell space-charge solver to examine the bunch dynamics in the whole interaction region of the gun. In order to focus on transient space-charge effects related to the finite bunch duration and exclude transients associated with the raise and fall of the external HV field applied to the cathode, we consider the limiting case of zero rise and fall time of the HV pulse, i.e., the electron bunch having a longitudinal square-box distribution on the cathode. The thermal emittance of the bunch is set to zero. The change in the emission current across the cathode-caused by the inhomogeneous applied electric field and the corresponding Schottky effect - is only $1.5 \%$ so that it is also not included into our simulations. The electrostatic field in the stripline-loop region calculated with the POISSON code is superimposed with the rf cavity field found with SUPERFISH to give the total field governing the dynamics of the electrons. As before, a $1 \mathrm{~ns}, 0.65 \mathrm{~A}$ electron bunch is first accelerated to $17.85 \mathrm{keV}$ by a $\mathrm{HV}$ pulse and then to $0.448 \mathrm{MeV}$ by a rf field.

The electron bunch distributions in the $r z$ plane of the gun at different time moments are depicted in Fig. 28. Though the outer electrons gain higher energies and travel faster through the gun, the fronts of bunch remains well defined and the electron density is quite uniform along the bunch except for the cathode region. The transverse phasespace of the slices of the bunch shown in Fig. 29 also replicates very well the one found earlier, cf. Fig. 26. The average total normalized slice emittance, including the thermal component, amounts to $0.32 \mathrm{~mm}$ mrad. This value is to be compared with the $0.3 \mathrm{~mm}$ mrad slice emittance found earlier using the decoupled simulations with EGUN and PARMELA. The total bunch emittance obtained with the fully time-dependent PARMELA simulations amounts only to $0.66 \mathrm{~mm}$ mrad compared with $0.6 \mathrm{~mm}$ mrad found earlier. However, it turned out that the best emittance compensation of the spherical aberration is now achieved with the $7.5 \mathrm{~mm}$ radius of curvature of the cathode instead of $5 \mathrm{~mm}$ found with EGUN. The transient space-charge 


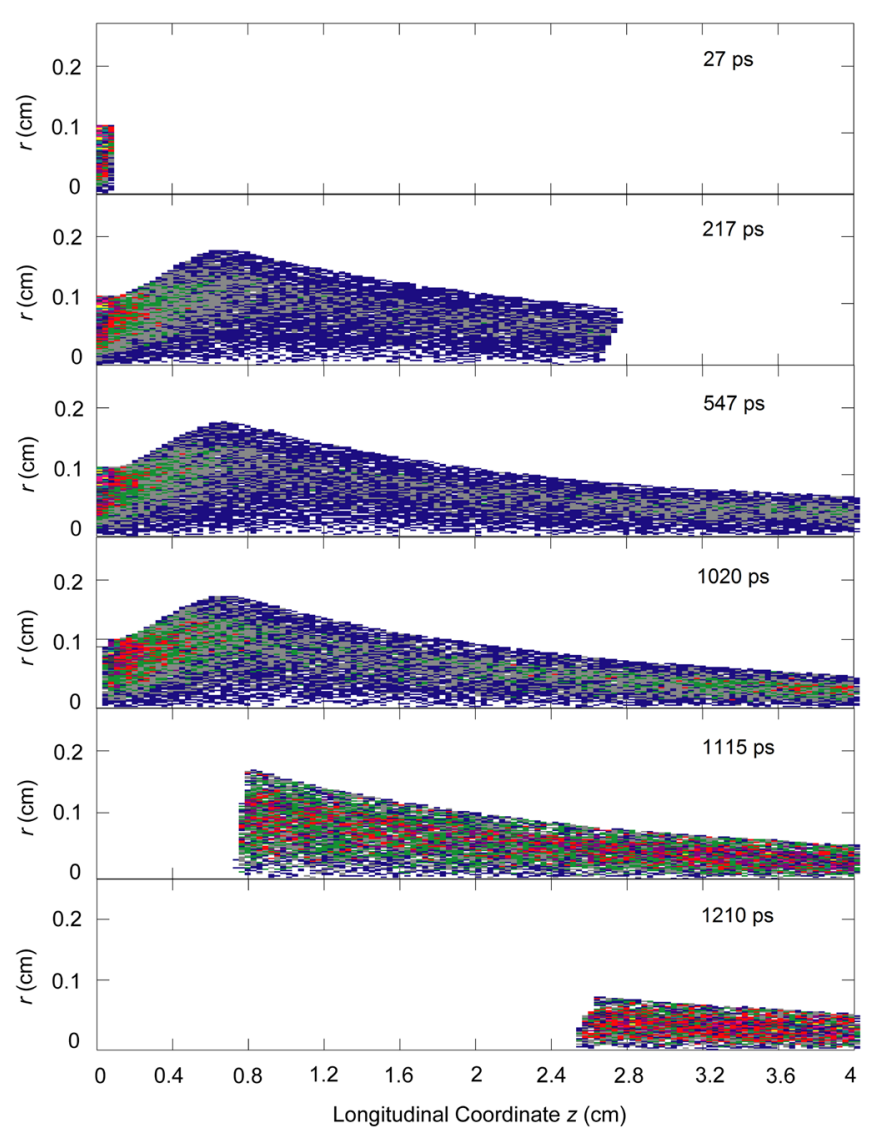

FIG. 28. Snapshots of the electron distributions in the $r z$ plane.

fields result in some acceleration of the leading slice of the bunch so that it gains about one $\mathrm{keV}$ higher energy compared to the previous EGUN-PARMELA simulations. Otherwise, the transient space-charge fields have no noticeable impact on the bunch dynamics. In overall, the fully time-dependent results are completely consistent with those obtained earlier using a combination of static and rfdependent approaches.

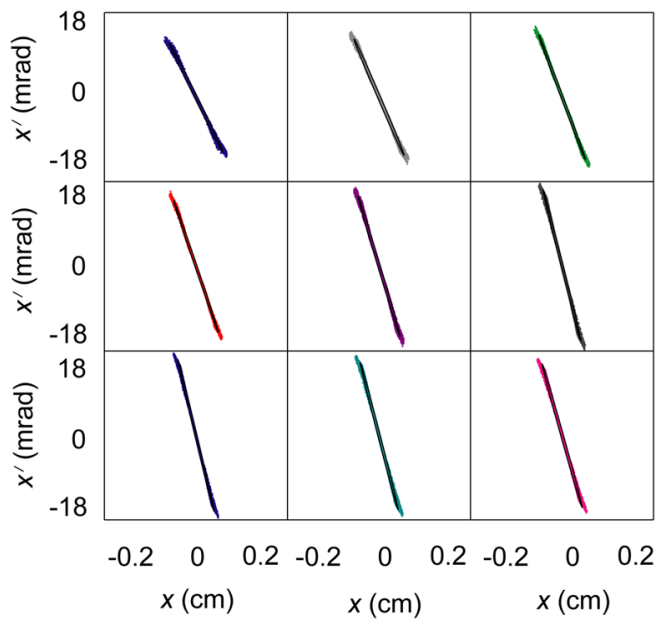

FIG. 29. Transverse phase-space of 9 consecutive slices without taking into account the initial thermal emittance.
In the present simulations we assumed a square-box HV pulse for gating the electron emission. However, in reality the profile of the HV pulse is more complicated and some electrons at the head and tail of the bunch will be accelerated to much lower energies in the stripline-loop configuration so that the beam optics becomes unmatched and the electrons acquire much larger transverse momenta. A study of the unmatched beam optics depends very much on the actual profile of the HV pulse and goes beyond the scope of the present paper. We anticipate that a bunch chopper might be needed to remove some leading and trailing electrons of the bunch to obtain a high-quality bunch emittance.

\section{CONCLUSION AND OUTLOOK}

We propose a thermionic cathode-based DC-rf gun that provides the bunch emittance at the same level as photocathode rf guns. The gun operates in a continuous-wave mode at a repetition rate up to $1 \mathrm{MHz}$. It is composed of a $10 \mathrm{MV} / \mathrm{m}$ DC diode integrated into a stripline-loop and a low-frequency $20 \mathrm{MV} / \mathrm{m}$ rf cavity. The beam optics is designed to keep the slice emittance of bunches as close to the thermal level as possible. A third harmonic cavity next to the gun is used to linearize the longitudinal phase-space of bunches and compensate for the phase-dependent bunch emittance growth in the rf gun cavity. The distinct features of our gun are: (i) nonlinear emittance compensation of spherical aberrations of the input rf cavity aperture, (ii) variable repetition rate, (iii) long lifetime of the cathode and iv) moderate requirements on vacuum. Our detailed design study indicates the possibility of generation of nC-scale bunches with an rms normalized slice and total bunch emittance of 0.32 and $0.66 \mathrm{~mm} \mathrm{mrad}$, respectively and an energy spread less than one $\mathrm{keV}$. The gun generates ns-long bunches, which requires additional bunch compression compared to photocathode rf guns. However, our preliminary study [41] shows that the generated bunches can be compressed down to several ps with nonlinear ballistic bunching involving harmonics of the frequency of a buncher. Or one can apply a compression scheme similar to the one used at the SACLA FEL since the bunch characteristics are very similar. To improve further the bunch emittance the leading and trailing electrons of the bunch can be cut away with a proper chopper.

The proposed combination of the DC HV diode and rf gun cavity results in a strong spherical aberration in the aperture-cavity region that severely degrades the bunch emittance. Hence, to counteract an S-shaped deformation of the bunch phase-space caused by the spherical aberration, we introduce a nonlinear Z-shaped distribution of transverse momenta of electrons before the aberration region by shaping the cathode. Thanks to this compensation, the slice emittance reaches a minimum of around $0.3 \mathrm{~mm}$ mrad for the design bunch 
current of 0.65 A. Since the design current corresponds to an extremum of the emittance, the emittance is current independent in the first order and a variation of the beam current by $50 \%$ around its design value yields only $6 \%$ increase in the beam emittance. Hence, the gun allows tunability in current.

The proposed gun is mainly intended for operation at a $100 \mathrm{kHz}$ repetition rate that is required for THz-pump$\mathrm{X}$-ray probe experiments. The results of simulations indicate that the bunch charge can be varied from around 450 to $850 \mathrm{pC}$ without sacrificing the emittance. Thus, the gun yields an average current from 45 to $85 \mu \mathrm{A}$. At the same time, the required HV pulser can also be operated up to one $\mathrm{MHz}$ repetition rate, resulting in an average current close to one $\mathrm{mA}$. Such a repetition rate is well suited to drive an X-ray FEL oscillator [22]. The power requirement on the pulser is around $5 \mathrm{~kW}$, which is still moderate.

\section{ACKNOWLEDGMENTS}

The authors acknowledge support from the StockholmUppsala Centre for Free Electron Laser Research. V.Z. acknowledges also support from the Swedish Research Council (VR). The authors are thankful to Dr. V. F. Zhiglo for fruitful discussions and grateful to Dr. K. Togawa for valuable comments and explanations, and for sharing the results of his simulations of the SACLA gun.

[1] W. A. Barletta and M. Borland, Report of the Basic Energy Sciences Workshop on Compact Light Sources (U.S. Dept. of Energy, Office of Science, Washington, 2010).

[2] T. Kubacka et al., Large-amplitude spin dynamics driven by a $\mathrm{THz}$ pulse in resonance with an electromagnon, Science 343, 1333 (2014).

[3] R. Schoenlein et al., SLAC Report No. SLACR-1053, 2015.

[4] C. Hernandez-Garcia et al., in Proceedings of the 21st Particle Accelerator Conference, Knoxville, TN, 2005 (IEEE, Piscataway, NJ, 2005), p. 3117.

[5] B. Dunham et al., in Proceedings of 2008 Linear Accelerator Conference, Victoria (TRIUMF, Vancouver, 2008).

[6] J. Grames et al., in Proceedings of the 21st Particle Accelerator Conference, Knoxville, TN, 2005 (IEEE, Piscataway, NJ, 2005), p. 2875.

[7] We are thankful to the referee for pointing this out.

[8] W. Kilpatrick, Criterion for vacuum sparking designed to include both rf and dc, Rev. Sci. Instrum. 28, 824 (1957).

[9] A. Arnold and J. Teichert, Overview on superconducting photoinjectors, Phys. Rev. ST Accel. Beams 14, 024801 (2011).

[10] D. H. Dowell et al., First operation of a photocathode radio frequency gun injector at high duty factor, Appl. Phys. Lett. 63, 2035 (1993).

[11] K. Baptiste, J. Corlett, S. Kwiatkowski, S. Lidia, J. Qiang, F. Sannibale, K. Sonnad, J. Staples, S. Virostek, and R.
Wells, A CW normal-conductive RF gun for free electron laser and energy recovery linac applications, Nucl. Instrum. Methods Phys. Res., Sect. A 599, 9 (2009).

[12] F. Sannibale et al., Advanced photoinjector experiment photogun commissioning results, Phys. Rev. ST Accel. Beams 15, 103501 (2012).

[13] J. Schmerge et al., in Proceedings of 36th International Free-Electron Laser Conference, Basel (Paul Scherrer Institut, Villigen, 2014).

[14] J. Madey, G. Ramian, and T. Smith, A fast pulsed electron gun system, IEEE Trans. Nucl. Sci. 27, 999 (1980).

[15] D. Oepts, A. van der Meer, and P. van Amersfoort, The free-electron-laser user facility FELIX, Infrared Phys. Technol. 36, 297 (1995).

[16] V. Bolotin et al., Status of the Novosibirsk terahertz FEL, Nucl. Instrum. Methods Phys. Res., Sect. A 543, 81 (2005).

[17] P. Sprangle, J. Peñano, B. Hafizi, D. Gordon, S. Gold, A. Ting, and C. Mitchell, High average current electron guns for high-power free electron lasers, Phys. Rev. ST Accel. Beams 14, 020702 (2011).

[18] K. Togawa, T. Shintake, T. Inagaki, K. Onoe, T. Tanaka, H. $\mathrm{Baba}$, and $\mathrm{H}$. Matsumoto, $\mathrm{CeB}_{6}$ electron gun for lowemittance injector, Phys. Rev. ST Accel. Beams 10, 020703 (2007).

[19] T. Shintake et al., Stable operation of a self-amplified spontaneous-emission free-electron laser in the extremely ultraviolet region, Phys. Rev. ST Accel. Beams 12, 070701 (2009).

[20] X. Dong et al., in Proceedings of the 25th International Linear Accelerator Conference, LINAC-2010, Tsukuba, Japan (KEK, Tsukuba, Japan, 2010).

[21] X. Dong et al., in Proceedings of the 24th Particle Accelerator Conference, PAC-2011, New York, 2011 (IEEE, New York, 2011).

[22] K.-J. Kim, Y. Shvyd'ko, and S. Reiche, A Proposal for an X-Ray Free-Electron Laser Oscillator with an EnergyRecovery Linac, Phys. Rev. Lett. 100, 244802 (2008).

[23] M. De Loos, S. Van der Geer, F. Kiewiet, O. Luiten, and M. van der Wiel, in Proceedings of the 8th European Particle Accelerator Conference, Paris, 2002 (EPS-IGA and CERN, Geneva, 2002), p. 1831.

[24] M. Reiser, Theory and Design of Charged Particle Beams (John Wiley \& Sons, New York, 2008).

[25] C.S. Hopper and J. R. Delayen, Superconducting spoke cavities for high-velocity applications, Phys. Rev. ST Accel. Beams 16, 102001 (2013).

[26] F. Sannibale, D. Filippetto, and C. F. Papadopoulos, Schemes and challenges for electron injectors operating in high repetition rate X-ray FELs, J. Mod. Opt. 58, 1419 (2011).

[27] I. V. Bazarov and C. K. Sinclair, Multivariate optimization of a high brightness dc gun photoinjector, Phys. Rev. ST Accel. Beams 8, 034202 (2005).

[28] Tech. Rep., GmbH, Darmstadt, Germany, 2009.

[29] L. Hu, J. Su, Z. Ding, Q. Hao, Y. Fan, and C. Liu, All solidstate high repetitive sub-nanosecond risetime pulse generator based on bulk gallium arsenide avalanche semiconductor switches, Rev. Sci. Instrum. 87, 086107 (2016). 
[30] Strictly speaking the scale of longitudinal localization must also depend on the size of inhomogeneity that is $r_{g}$ in our case but $r_{g} \sim h$ so we can try out a fitting formula that contains only, for example, $h$.

[31] Note that $\mu_{01}$ is simply the first root of $J_{0}[q]$ with $q$ being some generalized coordinate.

[32] A. Nassiri et al., in Proceedings of 48th ICFA Advanced Beam Dynamics Workshop on Future Light Sources (SLAC National Accelerator Laboratory, Menlo Park, 2010).

[33] J. H. Billen and L. M. Young, in Proceedings of the 15th Particle Accelerator Conference, PAC-1993, Washington, DC, 1993 (IEEE, New York, 1993), p. 790.

[34] W. Herrmannsfeldt, Stanford University SLAC Report No. 331, 1988.

[35] EGUN takes into account space-charge effects and boundary conditions on metallic surfaces.

[36] S. B. van der Geer, M. J. de Loos, J. I. M. Botman, O. J. Luiten, and M. J. van der Wiel, Nonlinear electrostatic emittance compensation in kA, fs electron bunches, Phys. Rev. E 65, 046501 (2002).

[37] A physically meaningful model consists of an infinitesimally small charged ball moving freely along an ideal rod. The rod is kept in place by an external nonelectromagnetic force and the energy $m v_{r}^{2} / 2$ is interpreted as the amount of work done by this force to balance the transverse momentum imparted by the electric field onto the ball.

[38] cas.web.cern.ch/cas/brunnen/presentations/pdf/Small\% 20Emittance\%20Sources.pdf.

[39] K.-J. Kim, Rf and space-charge effects in laser-driven rf electron guns, Nucl. Instrum. Methods Phys. Res., Sect. A 275, 201 (1989).

[40] T. I. Smith, in Proceedings of 1986 Particle Accelerator Conference (SLAC National Accelerator Laboratory, Menlo Park, 1986), p. 421.

[41] A. Opanasenko et al., in Proceedings of 5th Particle Accelerator Conference (2014), http://accelconf.web.cern .ch/AccelConf/IPAC2014/. 